
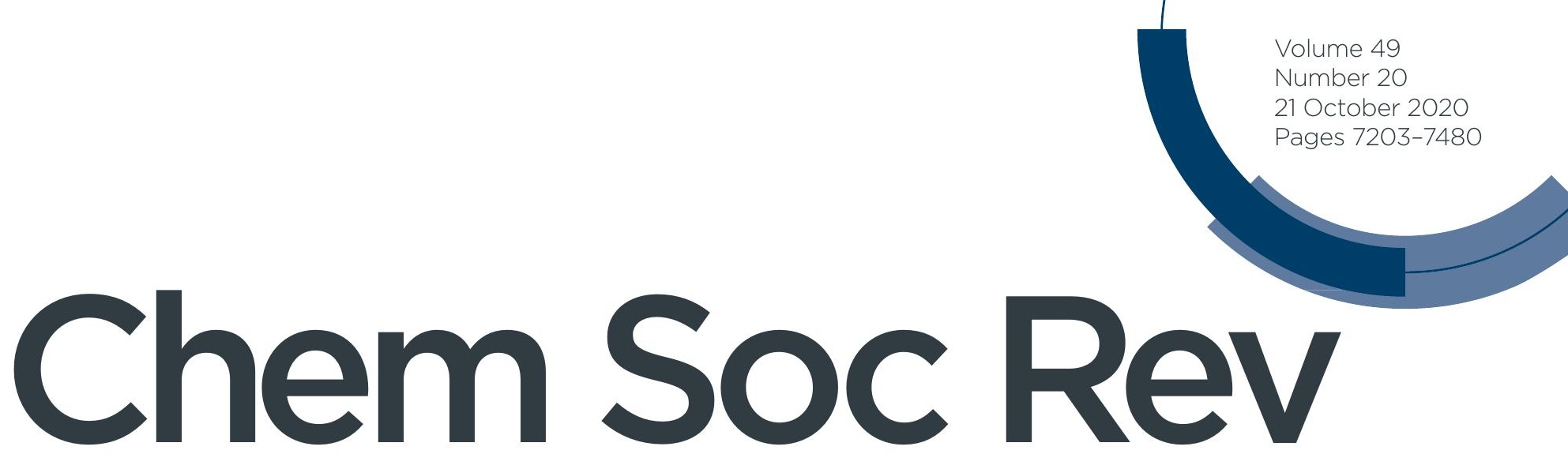

Chemical Society Reviews

rsc.li/chem-soc-rev

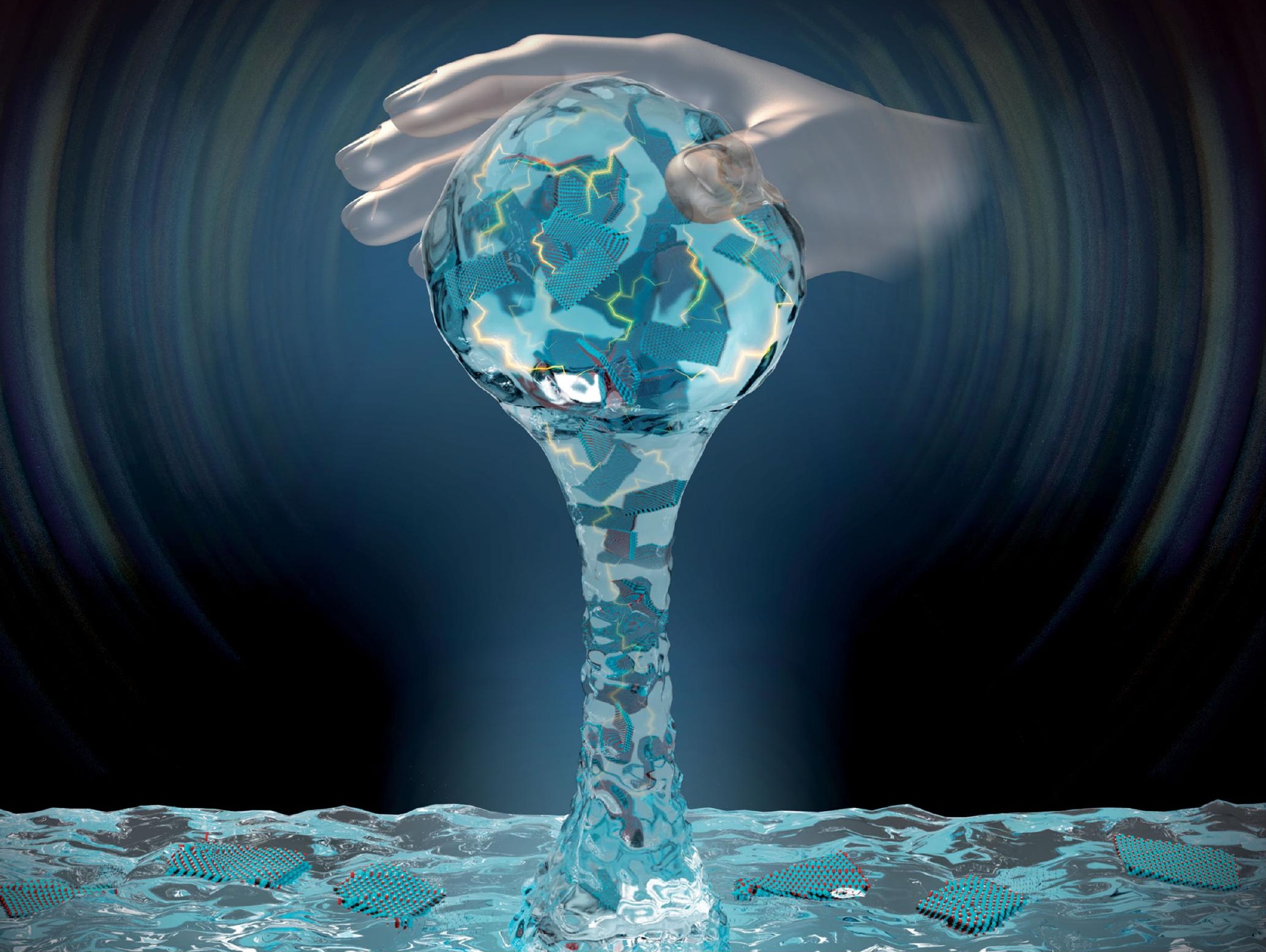

ISSN 0306-0012 
Check for updates

Cite this: Chem. Soc. Rev., 2020, 49, 7229

Received 29th April 2020

DOI: $10.1039 / \mathrm{d} 0 \operatorname{cs} 00022 a$

rsc.li/chem-soc-rev

\section{MXene hydrogels: fundamentals and applications}

\author{
Yi-Zhou Zhang, (D) $\dagger^{\mathrm{a}}$ Jehad K. El-Demellawi, (D) $\dagger^{\mathrm{a}}$ Qiu Jiang, ${ }^{\mathrm{a}}$ Gang Ge, ${ }^{\mathrm{ab}}$ \\ Hanfeng Liang, (D) ${ }^{a}$ Kanghyuck Lee, ${ }^{a}$ Xiaochen Dong (iD) ${ }^{\text {bc }}$ and Husam N. Alshareef (DD ${ }^{a}$
}

\begin{abstract}
Hydrogels have recently garnered tremendous interest due to their potential application in soft electronics, human-machine interfaces, sensors, actuators, and flexible energy storage. Benefiting from their impressive combination of hydrophilicity, metallic conductivity, high aspect ratio morphology, and widely tuneable properties, when two-dimensional (2D) transition metal carbides/nitrides (MXenes) are incorporated into hydrogel systems, they offer exciting and versatile platforms for the design of MXene-based soft materials with tunable application-specific properties. The intriguing and, in some cases, unique properties of MXene hydrogels are governed by complex gel structures and gelation mechanisms, which require in-depth investigation and engineering at the nanoscale. On the other hand, the formulation of MXenes into hydrogels can significantly increase the stability of MXenes, which is often the limiting factor for many MXene-based applications. Moreover, through simple treatments, derivatives of MXene hydrogels, such as aerogels, can be obtained, further expanding their versatility. This tutorial review intends to show the enormous potential of MXene hydrogels in expanding the application range of both hydrogels and MXenes, as well as increasing the performance of MXene-based devices. We elucidate the existing structures of various MXene-containing hydrogel systems along with their gelation mechanisms and the interconnecting driving forces. We then discuss their distinctive properties stemming from the integration of MXenes into hydrogels, which have revealed an enhanced performance, compared to either MXenes or hydrogels alone, in many applications (energy storage/ harvesting, biomedicine, catalysis, electromagnetic interference shielding, and sensing).
\end{abstract}

\section{Key learning points}

(1) Hydrogels made of MXenes find wide applications such as energy storage, electronics, sensing, catalysis, and drug delivery.

(2) Understanding the possible gelation mechanisms of several MXene-based hydrogels.

(3) MXenes can act as gelators, initiators, crosslinkers, and multifunctional nanofillers during the formation of hydrogels.

(4) MXenes can bring about both improved mechanical properties and exciting new functionalities when incorporated into hydrogels.

(5) Future perspectives of the endless opportunities awaiting MXene hydrogels and their derivatives.

\section{Introduction}

Standing at the dawn of the $5 \mathrm{G}$ era, we are about to witness dramatic changes in our everyday experience in an unprecedented way. Wearable electronics such as physiological sensors,

\footnotetext{
${ }^{a}$ Physical Science and Engineering Division, Materials Science \& Engineering, King Abdullah University of Science and Technology (KAUST), Thuwal 23955-6900, Kingdom of Saudi Arabia. E-mail: husam.alshareef@kaust.edu.sa

${ }^{b}$ Key Laboratory of Flexible Electronics (KLOFE) and Institute of Advanced Materials (IAM), School of Physical and Mathematical Sciences, Nanjing Tech University (NanjingTech), Nanjing 211800, China School of Chemistry and Materials Science, Nanjing University of Information Science and Technology, Nanjing 210044, China

$\dagger$ These authors contributed equally.
}

bioelectronic interfaces, and robotic prostheses are expecting booming development. ${ }^{1}$ However, one major hurdle facing these flexible and stretchable electronics is the mismatch between human tissues and traditional electronics in terms of both mechanical and electrical properties, i.e., human tissues are soft, and conduct electric signals through ions, whereas electronics are rigid and rely on electrons to conduct electricity. In this context, considerable attention has been drawn to natureinspired materials such as hydrogels, a class of intrinsically stretchable ionic conductors that are compatible with human tissues both mechanically and electrically. They have already shown great promise in various flexible 'ionotronic' devices where both ions and electronics contribute to the functioning of many devices such as artificial muscles/skins/axons and 
stretchable energy storage devices. ${ }^{2,3}$ Nowadays, we are witnessing a broader interest in employing hydrogels in numerous fields beyond the traditional biomedical sector. Due to the recent improvement of their comprehensive performance in terms of mechanical, chemical, and electrical properties, hydrogels can act as a better bridge between biology and electronics.

One of the fascinating aspects of hydrogels is that they are primarily composed of water $(>99 \%)$ along with the solvated molecules/polymers/inorganic particles (gelators); meaning that they can maintain the same physicochemical properties as in liquid water while exhibiting a macroscopically solid-like rheological behavior. ${ }^{4}$ They are also excellent precursors to other useful functional mateirals, e.g., aerogels and xerogels, ${ }^{5,6}$ that are typically referred to as hydrogel derivatives. The threedimensional (3D) networks of hydrogels or any of their derivatives are generally governed by an ensemble of several chemical (covalent) and physical (e.g., hydrophobic and electrostatic) interactions, and they are mostly made of polymeric chains. Other gelators such as $2 \mathrm{D}$ materials have also been reported to be used in the presence/absence of polymeric networks. Their involvement has long been demonstrated as an effective approach to improve the characteristics of the formed hydrogels. The presence of these nanomaterials not only enhances the mechanical properties of hydrogels but also offers new multifunctional

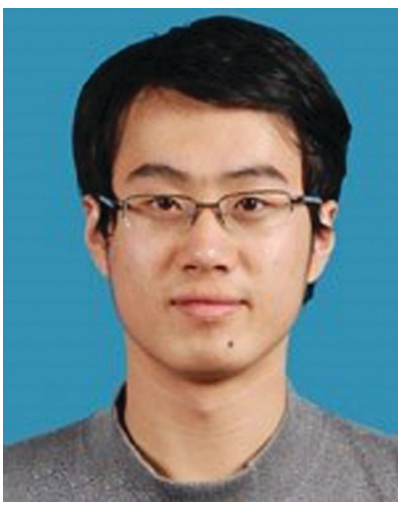

Yi-Zhou Zhang
Dr Yi-Zhou Zhang received his Bachelor's degree from Nanjing University, after which he obtained a PhD from Nanjing University of Posts \& Telecommunications under the supervision of Prof. Wei Huang. $\mathrm{He}$ is currently a postdoctoral fellow in Prof. Husam $N$. Alshareef's group at KAUST. His present research primarily focuses on exploring MXenes for new applications. responsiveness (e.g., electrical, optical, thermal, acoustic, magnetic, etc.).

Among the existing 2D nanomaterials, the thriving family of transition metal carbides, nitrides, or carbonitrides (MXenes) stand out for their unique combination of metallic conductivity, solution processability, high-aspect-ratio, and widely tunable properties. $^{7-10}$ Generally, MXenes have the chemical formula $\mathrm{M}_{n+1} \mathrm{X}_{n} \mathrm{~T}_{x}(n=1-4)$, where $\mathrm{M}$ and $\mathrm{X}$ stand for early transition metals (e.g., Ti, V, Nb, Mo, etc.), and carbon and/or nitrogen, respectively. While $\mathrm{T}_{x}$ denotes different populations of surfaceterminated groups (e.g., OH, O, and/or F). Thus far, MXenes have already demonstrated very good performance in a gamut of applications spanning energy storage, sensing, optoelectronics, catalysis, and biomedicine., ${ }^{71-13}$ Furthermore, concerning hydrogel-based applications, MXenes are of particular interest owing to their excellent mechanical strength, ${ }^{14}$ exceptional hydrophilicity, ${ }^{8,15}$ and rich surface chemistry that provides another level of versatility. ${ }^{10}$ Thus, when MXenes are incorporated in hydrogel systems, they bring about both improved properties and exciting novel functionalities for enhanced performances in various applications. The distinctive new characteristics of the obtained MXene-based gels either arise from the inherent features of the MXenes, the summation of the functionalities of both MXenes and other components in the gel matrix, or even evolve from the synergistic interactions between them. Indeed, the formulation of MXenes into hydrogels not only allows for the design of MXene-based soft materials with tailorable properties but also significantly increases the stability of MXenes, which is often a limiting factor for many of their applications. Moreover, through simple treatments, other MXene hydrogel derivatives such as aerogels can be prepared, further expanding the versatility of their applications. It is noteworthy that in the past two years, there was an exponential growth in high-quality publications regarding MXene hydrogels and their derivatives, turning it into a highly competitive field of research.,6,13,16-19 However, given the nascent stage of research on MXenes and its large ever-expanding family, endless possibilities await, either in terms of potential exciting applications, or new mechanisms for MXene-based hydrogel formation and subsequent improvement in the performances.

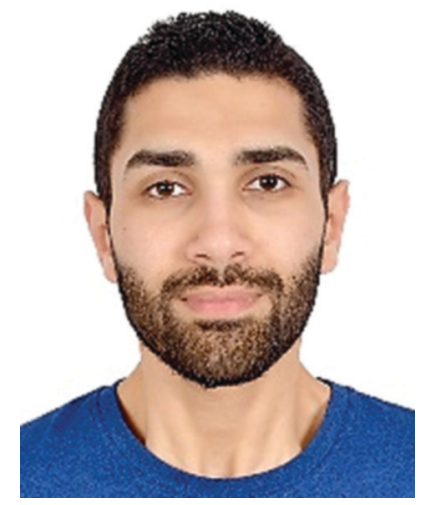

Jehad K. El-Demellawi
Jehad K. El-Demellawi is a PhD candidate in Materials Science \& Engineering under the supervision of Prof. Husam N. Alshareef at KAUST. His current research focuses on the synthesis and characterization of various MXenes with novel properties for different applications.

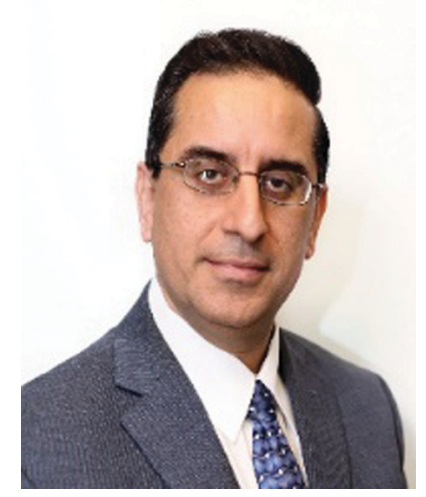

Husam N. Alshareef is a Professor of Materials Science \& Engineering at KAUST. After nearly ten years in the semiconductor industry, he joined KAUST in 2009 where he initiated an active research group working on semiconductor nanomaterial development for energy storage and electronics. 
Hence, this timely review is intended to promote the development of such emerging systems and expand the application range of both hydrogels and MXenes. To our knowledge, a review focusing on MXene hydrogels and their derivatives has not been reported.

In the following sections, we discuss the synthesis protocols of various MXene-based gels while elucidating the underlying gelation mechanisms as well as the governing structure-property relationships in light of the exotic combination of excellent electrochemical, mechanical, and optoelectronic properties of MXenes. We then shed light on the versatile applications of MXene-based gels, including energy storage, biomedicine, catalysis, electromagnetic interference (EMI) shielding, sensing, and energy harvesting. Finally, we underline the main challenges and potential research directions for MXene hydrogels and their derivatives.

\section{Synthesis and gelation mechanisms of MXene hydrogels and their derivatives}

Hitherto, all reported MXene-containing gels have been made of $\mathrm{Ti}_{3} \mathrm{C}_{2} \mathrm{~T}_{x}$, i.e., the most studied MXene among the 25+ MXenes reported to date, ${ }^{7,20}$ except for one case where $\mathrm{Ti}_{2} \mathrm{CT}_{x}$ was used to construct one of the hydrogel derivatives - cryogel. $^{21}$ Following previous reports, ${ }^{20}$ all the MXene nanosheets, contained in the MXene-based gels reviewed here were synthesized by selectively removing the "A" layers (i.e., mostly groups 13 and 14 elements) from their parent layered 3D MAX phases, using different top-down fluoride-containing etching methods. MAX phases can be described as alternately stacked "MX" layers and close-packed "A" atomic layers, with a general formula of $\mathrm{M}_{n+1} \mathrm{AX}_{n}$, where the $\mathrm{M}-\mathrm{X}$ ionic/covalent bonds are chemically more stable than the $\mathrm{M}-\mathrm{A}$ metallic bonds. The exfoliation of those stacked layers is attained by the selective etching of the "A" layers, forming stacks of multilayered
MXenes terminated by different functional groups (i.e., $\mathrm{T}_{x}$ ). For single and few-layered MXenes, a subsequent delamination process is essential to open up the interlayer spacing by intercalating different cations $\left(\right.$ e.g., $\mathrm{Li}^{+}$) between the A-depleted multilayered MXenes. As an example, the general synthesis pathway of $\mathrm{Ti}_{3} \mathrm{C}_{2} \mathrm{~T}_{x}$ is schematically represented in Fig. $1 .^{20}$

Furthermore, even with the predominance of $\mathrm{Ti}_{3} \mathrm{C}_{2} \mathrm{~T}_{x}$ in MXene hydrogels and their derivatives, its role and contribution to the gelation process distinctively vary from one study to another. In principle, the continuous phase in a typical MXene gel (e.g., water in the case of hydrogels) would be confined within a 3D structured network that is physically and/or chemically crosslinked by a secondary phase, i.e., the gelator. Up till now, the reported gelators for MXene hydrogels are either MXenes themselves, reduced graphene oxide (rGO), polymers, inorganic ions, or combinations of any of them. Thus, for ease of discussion, we will classify the synthetic routes for the MXene-containing hydrogels based on the gelators involved. Fig. 2 depicts four different structural illustrations for the reported MXene hydrogels. The nature of the interactions between the MXene nanosheets network in the presence/absence of other gelators within the hydrogel can vary depending on the driving forces involved in the gelation process. These driving forces, either physical or chemical, are responsible for assembling the 3D MXene network of the hydrogel or any of its derivatives and maintaining it intact.

\subsection{All-MXene hydrogels}

Given the inevitable aggregation and restacking tendency of MXene nanosheets as a result of the sturdy van der Waals interlayer attractions, it is difficult for MXenes to form a gel on their own. It is even more challenging in the case of hydrogels, owing to the superior hydrophilicity induced by the surface moieties of MXene. ${ }^{7,15}$ For this reason, it is often essential to have another component (i.e., crosslinker) in the hydrogel matrix that can counterbalance the hydrophilicity of MXenes and maintain the 3D assembly of the $2 \mathrm{D}$ nanosheets. However,

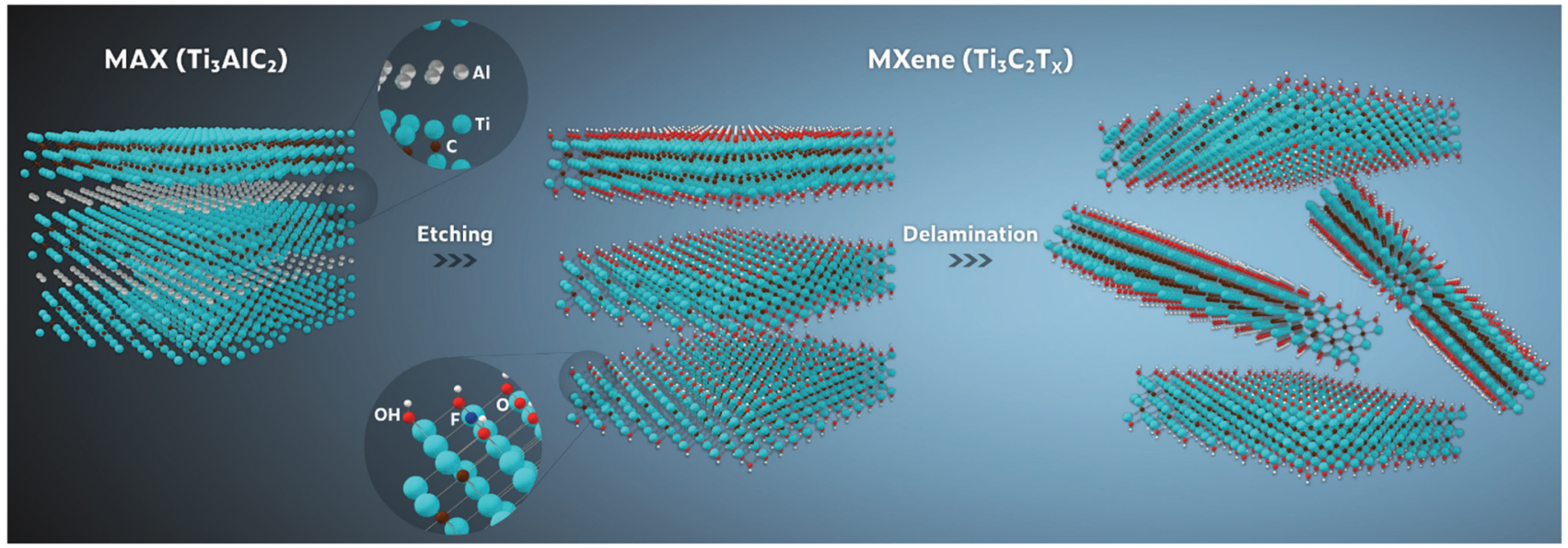

Fig. 1 General synthesis pathway of $\mathrm{Ti}_{3} \mathrm{C}_{2} \mathrm{~T}_{x}$ MXene nanosheets, using fluorine-based top-down etching methods. (left) Parent layered Ti ${ }_{3} A l C_{2} M A X$ phase, (middle) exfoliated (etched), and (right) delaminated MXene nanosheets. 


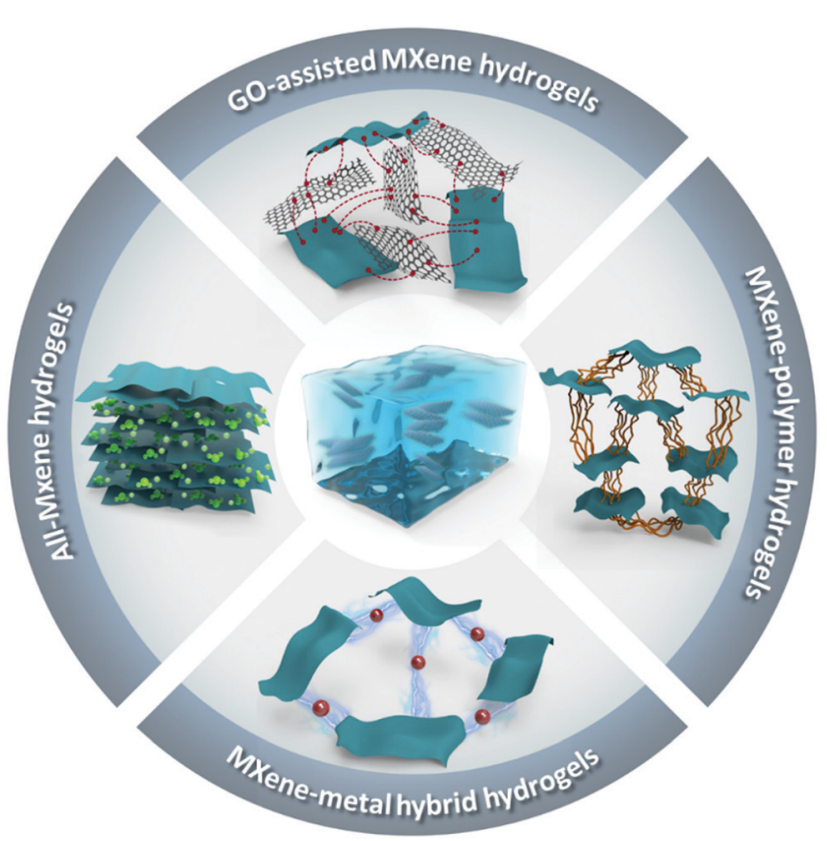

Fig. 2 Schematic illustrations of the reported MXene-containing hydrogels. Clockwise: all-MXene, GO-assisted MXene, MXene-polymer, and MXenemetal hybrid nanocomposite hydrogels.

despite all the above challenges, Lin et al. and Lukatskaya et al. have succeeded in preparing all-MXene hydrogels, with $\mathrm{Ti}_{3} \mathrm{C}_{2} \mathrm{~T}_{x}$ being the only gelator involved, by using vacuum-assisted filtration of delaminated $\mathrm{Ti}_{3} \mathrm{C}_{2} \mathrm{~T}_{x}$ suspensions. ${ }^{22,23}$ Although this is a very common method for making MXene 'paper' films, they managed to attain a $\mathrm{Ti}_{3} \mathrm{C}_{2} \mathrm{~T}_{x}$ hydrogel by immediately disconnecting the vacuum as soon as no colloidal solution was left on the porous membrane. A schematic of the filtration process is displayed in Fig. 3a. The hydrogel film was then briefly dipped into acetone to facilitate its peeling off. The substantial presence of the unremoved pre-intercalated water molecules has induced a dynamical physical crosslinking between the MXene sheets, which was responsible for the gel-like texture. However, this kind of force cannot sustain the prevailing van der Waals interlayer forces. Thus, to prevent the restacking of the MXene nanosheets, each of the abovementioned studies followed a different strategy to avoid the collapse of the formed hydrogels. On the one hand, Lin et al. simply immersed the non-dried hydrogel film in a thermally stable ionic liquid, i.e., 1-ethyl-3-methylimidazolium bis(trifluoromethylsulfonyl)imide (EMI-TFSI), to allow for solvent exchange. After vacuum drying at $80{ }^{\circ} \mathrm{C}$, the ionic liquid remained in the MXene film, which prevented the restacking and increased the interlayer spacing, forming a $\mathrm{Ti}_{3} \mathrm{C}_{2} \mathrm{~T}_{x}$ ionogel instead of a hydrogel. In the other study, Lukatskaya et al. preserved the structure of the obtained MXene hydrogel by soaking it in an $\mathrm{H}_{2} \mathrm{SO}_{4}$ electrolyte for three days, after which it was directly used for electrochemical measurements. Fig. 3b demonstrates the MXene structure with the pre-intercalated water molecules, which enables the fast transportation of protons. ${ }^{23,24}$ a

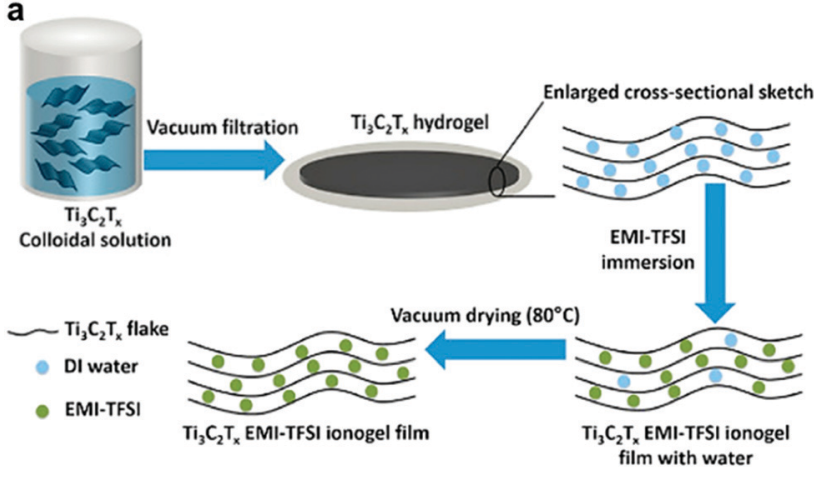

b

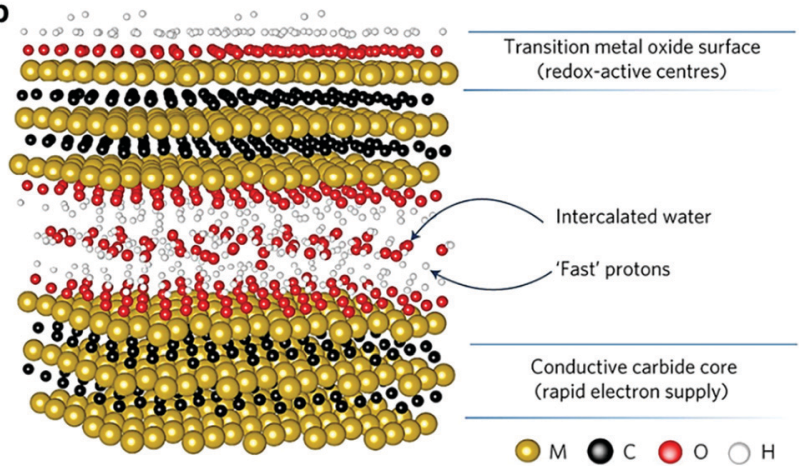

Fig. 3 (a) Schematic illustration of all-MXene hydrogel and ionogel formation. Reproduced with permission from ref. 22 (copyright 2016, Elsevier). (b) Structure of the MXene nanosheets with pre-intercalated water molecules facilitating the fast transport of protons to the redoxactive centers. Reproduced with permission from ref. 23 (copyright 2017, Nature Publishing Group).

\subsection{GO-assisted MXene nanocomposite hydrogels}

In addition to the abovementioned physically-derived gelation mechanism, other physical and chemical interactions can also assist in the formation of MXene hydrogels and allow for additionally reinforced skeletons. The ability of crosslinkers to assemble freestanding MXene nanosheets suspended in water into a well-connected $3 \mathrm{D}$ structure is crucial for the gelation process. However, the selection of a suitable crosslinker (gelator) remains challenging owing to the limited active crosslinking sites on the MXene surfaces. ${ }^{15,25}$ Therefore, the exposure of more accessible crosslinking sites is essential to obtain a well-assembled hydrogel network. This could be achieved with the assistance of another 2D material-based gelator - GO, which would allow for interfacial interactions with the MXene nanosheets rather than a point-to-plane interaction. Such an intimate interfacial crosslinking between $\mathrm{Ti}_{3} \mathrm{C}_{2} \mathrm{~T}_{x}$ and $\mathrm{rGO}$ nanosheets was first reported by Chen et al. via an organics-free self-convergence process. ${ }^{26}$ When mixed with GO solution, $\mathrm{Ti}_{3} \mathrm{C}_{2} \mathrm{~T}_{x}$ was able to reduce the hydrophilic GO to a more hydrophobic rGO as a result of the partial removal of the abundant oxygen-containing surface species on the surface of the GO sheets (Fig. 4a). The remarkable ability of $\mathrm{Ti}_{3} \mathrm{C}_{2} \mathrm{~T}_{x}$ to act as a reducing agent is attributed to the multivalent nature of its $\mathrm{Ti}$ species that can transform from lowvalence into surface-terminated high-valence states. During the 
a
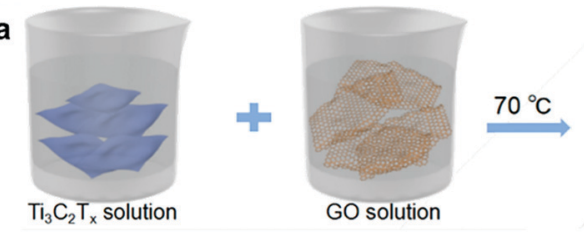

b
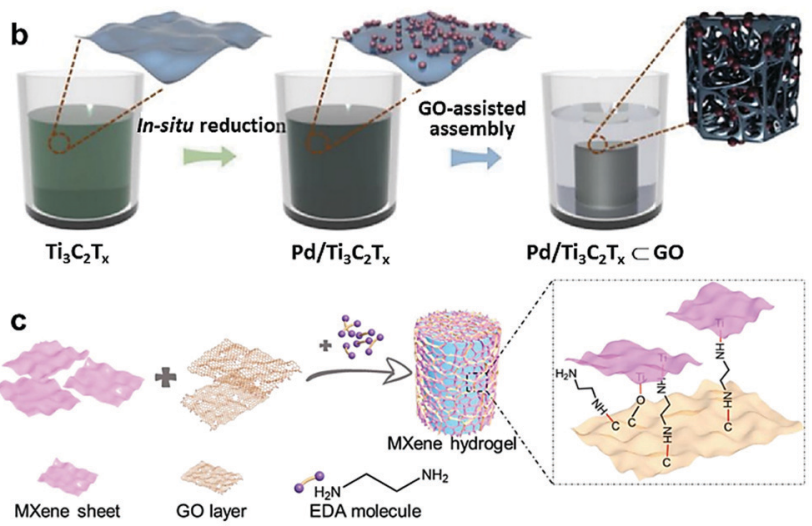

Fig. 4 Schematic illustrations of GO-assisted gelation for various MXene hydrogels. (a) $\mathrm{Ti}_{3} \mathrm{C}_{2} \mathrm{~T}_{x}-\mathrm{rGO}$ hydrogel. Reproduced with permission from ref. 26 (copyright 2018, American Chemical Society). (b) Pd-Decorated $\mathrm{Ti}_{3} \mathrm{C}_{2} \mathrm{~T}_{x}-\mathrm{rGO}$ hydrogel. Reproduced with permission from ref. 27 (copyright 2020, Elsevier). (c) An EDA-initiated $\mathrm{Ti}_{3} \mathrm{C}_{2} \mathrm{~T}_{x}-\mathrm{rGO}$ hydrogel. Reproduced with permission from ref. 29 (copyright 2019, Wiley-VCH).

gelation process, the reduced electrostatic repulsion between $\mathrm{Ti}_{3} \mathrm{C}_{2} \mathrm{~T}_{x}$ and rGO facilitates the self-convergence of the MXene nanosheets into the anisotropically-assembled rGO framework, driven by the hydrogen bonding between $\mathrm{Ti}_{3} \mathrm{C}_{2} \mathrm{~T}_{x}$ and $\mathrm{rGO}^{26}$ Analogous GO-assisted interfacial crosslinking was also used to fabricate Pd-decorated $\mathrm{Ti}_{3} \mathrm{C}_{2} \mathrm{~T}_{x}$ hydrogel by utilizing the redox capability of $\mathrm{Ti}_{3} \mathrm{C}_{2} \mathrm{~T}_{x}$ in reducing Pd ions to metallic Pd, which were gradually deposited on the MXene sheets (Fig. 4b). ${ }^{27,28}$

To further increase the accessible surface area during the GO-assisted gelation process, Shang et al. introduced an interlayer spacer, ethylenediamine (EDA), that led to the formation of chemical bonding between rGO layers and MXene nanosheets (Fig. 4c). ${ }^{29}$ Simultaneous to the $\mathrm{Ti}_{3} \mathrm{C}_{2} \mathrm{~T}_{x}$-induced reduction of GO, the EDA promoted the formation of oxygen dangling bonds by opening the epoxy rings present on the GO sheets. $\mathrm{Ti}_{3} \mathrm{C}_{2} \mathrm{~T}_{x}$ then connected with these dangling bonds forming MXene-rGO hybrid structures that turned into hydrogels by the spontaneous interlayer attraction forces between the hybrid nanosheets. Compared to GO-only hydrogels, MXene-rGO nanocomposite (NC) hydrogels are thicker and more flexible. ${ }^{29}$ It should also be noted that the introduction of EDA as a spacer has reduced the critical gelation concentration (CGC) of $\mathrm{Ti}_{3} \mathrm{C}_{2} \mathrm{~T}_{x}$ required to form a MXene-rGO NC hydrogel.

\subsection{MXene-polymer nanocomposite hydrogels}

On account of their hydrophilicity, the incorporation of MXene nanosheets into polymer hydrogel networks, which are extensively swollen with water, has endowed them with outstanding multifunctional versatility. To date, several MXene-polymer
NC hydrogels were prepared and exploited in many applications. ${ }^{13,18,19,30-34}$ Typically, the interactions between MXene nanosheets and other polymers in the hydrogel network arise from polymer chain entanglements, ionic interactions, hydrogen and/or covalent bonding. However, the role of MXene nanosheets in the gelation process of the reported MXene-polymer NC hydrogels varies substantially from noninvolvement to initiating the gelation or acting as a crosslinker. In principle, analogous to 'classical' polymer hydrogels, three integral components are needed to synthesize MXene-polymer NC hydrogels, i.e., monomer, initiator, and a crosslinker. During the gelation-accompanied polymerization step, if a single monomer is involved, then the $3 \mathrm{D}$ assembled networks are referred to as MXene-homopolymeric NC hydrogels. Whereas crosslinked networks derived from two or more different monomer species in the presence of MXene nanosheets are referred to as MXene-copolymeric NC hydrogels. For instance, Liao et al. fabricated MXene-copolymeric hydrogels by dissolving acrylamide (AAm) monomers and polyvinyl alcohol (PVA) in water containing $\mathrm{Ti}_{3} \mathrm{C}_{2} \mathrm{~T}_{x}$ nanosheets (Fig. 5a). ${ }^{18}$ Sodium tetraborate decahydrate (borax) was used as a dynamic crosslinker between the PVA chains, where the $-\mathrm{OH}$ groups were interconnected by tetrafunctional borate $\left(\mathrm{B}(\mathrm{OH})_{4}{ }^{-}\right)$ions. The polyacrylamide (PAAm) network was subsequently obtained through the in situ polymerization of the AAm monomers at $60{ }^{\circ} \mathrm{C}$. During the gel formation, MXene nanosheets were successfully incorporated into the hydrogel network as a result of the polymeric chain entanglements, where it served as another crosslinker via supramolecular interactions such as hydrogen bonds, between PVA and the hydrophilic surface moieties of the MXene nanosheets. In parallel, the hydroxyl groups of $\mathrm{Ti}_{3} \mathrm{C}_{2} \mathrm{~T}_{x}$ were also covalently bonded with the $\mathrm{B}(\mathrm{OH})_{4}{ }^{-}$ ions. Following the formation of the $\mathrm{Ti}_{3} \mathrm{C}_{2} \mathrm{~T}_{x}-\mathrm{PAAm}-\mathrm{PVA} \mathrm{NC}$ hydrogel, an organohydrogel of the same NC was obtained by simple solvent displacement as will be discussed in Section 2.5.

On the other hand, different MXene-homopolymeric NC hydrogels were prepared using several polymers, e.g., PVA, cellulose, chitosan, and acrylate polymers. For instance, we used a simple preparation method to obtain MXene-PVA NC hydrogels. ${ }^{13,19}$ In a typical experiment, $\mathrm{Ti}_{3} \mathrm{C}_{2} \mathrm{~T}_{x}$ paste (sediment) obtained by centrifuging MXene suspensions was spread on a commercially available PVA hydrogel, then hand-rolled and flattened for a few times until a uniformly black MXene-PVA hydrogel was attained. The incorporation of $\mathrm{Ti}_{3} \mathrm{C}_{2} \mathrm{~T}_{x}$ into the PVA network was enabled by the hydrogen bonding between the abundant hydrophilic surface moieties of the MXene and the hydroxyl groups of PVA. Another $\mathrm{Ti}_{3} \mathrm{C}_{2} \mathrm{~T}_{x}$-PVA hydrogel was obtained through the direct dispersion of MXene nanosheets into the PVA aqueous solution (Fig. 5b). ${ }^{33}$ To assist in the gelation process, sodium tetraborate solution was added to the mixture as a crosslinking agent, forming di-diol bonds between the functional groups of the PVA chains. During the gelation, the oxygen-containing surface groups of $\mathrm{Ti}_{3} \mathrm{C}_{2} \mathrm{~T}_{x}$ covalently bond with the $\mathrm{B}(\mathrm{OH})_{4}{ }^{-}$ions and the PVA chains. Furthermore, another MXene-based homopolymer NC hydrogel was fabricated using cellulose as a crosslinker interconnecting 
a

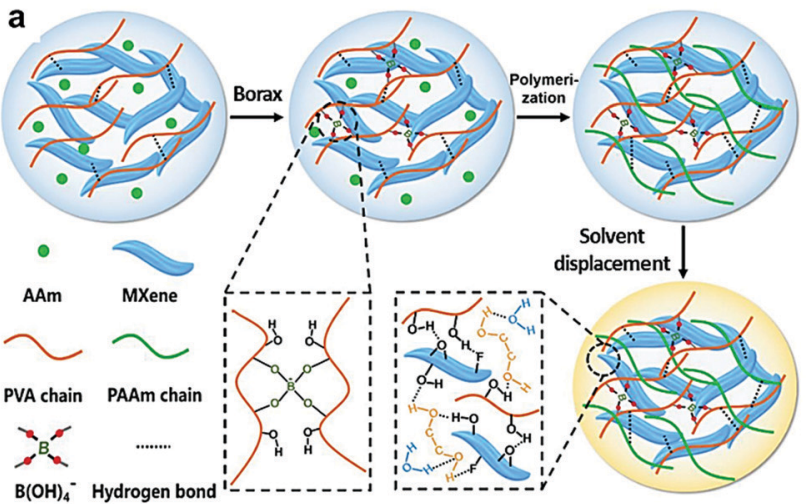

b

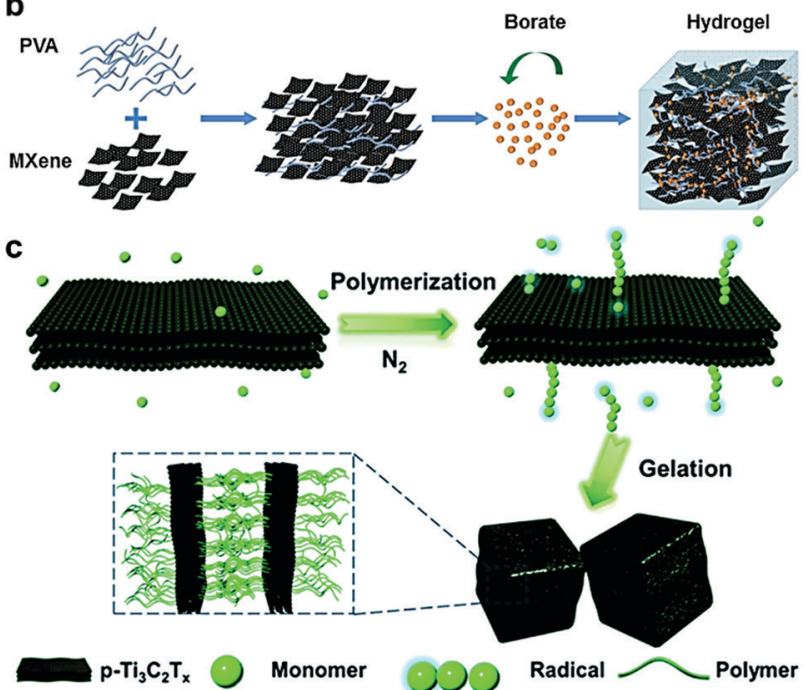

(M) monomer

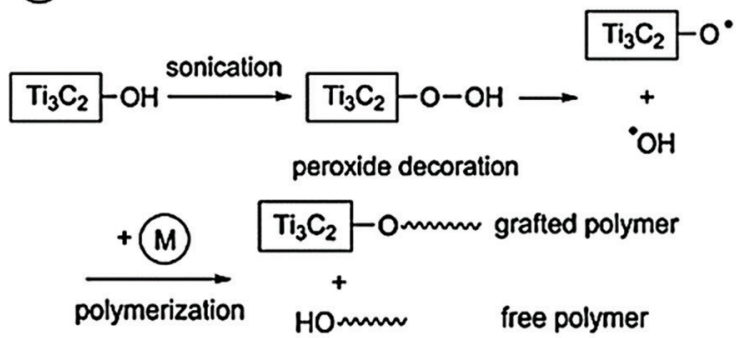

Fig. 5 Schematic illustrations showing the synthesis of polymeric MXene hydrogels. (a) Synthesis of MXene-PAAm-PVA NC hydrogel. Reproduced with permission from ref. 18 (copyright 2019, Wiley-VCH). (b) Synthesis of MXene-PVA NC hydrogel. Reproduced with permission from ref. 33 (copyright 2019, Wiley-VCH). (c) Peroxide-decorated $\mathrm{Ti}_{3} \mathrm{C}_{2} \mathrm{~T}_{x}$-initiated polymerization mechanism and gelation of different MXene-polymer NC hydrogels. Reproduced with permission from ref. 32 (copyright 2019, Royal Society of Chemistry).

the MXene nanosheets with hydrogen and covalent bonding. ${ }^{30}$ To prepare MXene-cellulose NC hydrogels, Xing et al. mixed $\mathrm{Ti}_{3} \mathrm{C}_{2} \mathrm{~T}_{x}$ nanosheets suspended in water with an as-prepared cellulose solution in the presence of epichlorohydrin (ECH) as a crosslinker. The incorporation of the MXene nanosheets did not interrupt the crosslinking reaction of the cellulose chains with ECH, allowing for the formation of well-structured $\mathrm{Ti}_{3} \mathrm{C}_{2} \mathrm{~T}_{x}-$ cellulose hydrogels. Moreover, owing to its biocompatibility,
MXene nanosheets were mixed with preformed hydrogel solution of chitosan, i.e., an auspicious biopolymer, in the presence of honey to form a sweet $\mathrm{Ti}_{3} \mathrm{C}_{2} \mathrm{~T}_{x}$-chitosan NC hydrogel. ${ }^{34}$

In all the aforementioned reports, MXene nanosheets were mainly used as the multifunctional nanofiller to endow the host polymer hydrogel with specific properties. Nonetheless, their contribution to the gelation of the MXene-polymer hydrogel remains to be marginal. Recently, Tao et al. demonstrated the use of MXene nanosheets as an initiator for the polymerization and the gelation of several acrylic monomers to form different MXene-polyacrylate NC hydrogels. ${ }^{32}$ The polymerization of a series of monomers including acrylamide, $N$-isopropylacrylamide (NIPAM), N,N-dimethylacrylamide (DMA), methyl methacrylate (MMA), and hydroxyethyl methacrylate (HEMA), were initiated by peroxide-decorated MXene $\left(\mathrm{p}-\mathrm{Ti}_{3} \mathrm{C}_{2} \mathrm{~T}_{x}\right)$ nanosheets. The $\mathrm{p}-\mathrm{Ti}_{3} \mathrm{C}_{2} \mathrm{~T}_{x}$ nanosheets were obtained through a sonication-assisted etching process. The polymerization and the subsequent gelation of the corresponding MXene-polymer NC hydrogels occurred within a few minutes (ca. 5 minutes) under deoxygenated conditions (Fig. $5 \mathrm{c}$ ). It is worth mentioning that $\mathrm{Ti}_{3} \mathrm{C}_{2} \mathrm{~T}_{x}$ (obtained with no sonication) could not initiate the polymerization, which highlights the role of the peroxyl species bound to the surface of the $\mathrm{p}-\mathrm{Ti}_{3} \mathrm{C}_{2} \mathrm{~T}_{x}$ nanosheets in the polymerization of acrylic monomers. The crosslinking of the formed hydrogel networks was cooperatively achieved by hydrogen bonding, polymer chain entanglements, and hydrophobic interactions induced by the MXene nanosheets grafted with the polymer. The steric stability of the covalently grafted polymers has endowed the $\mathrm{Ti}_{3} \mathrm{C}_{2} \mathrm{~T}_{x}$-polymer NC hydrogel with high stability. In addition to its ability to initiate the gelation process, MXene nanosheets were recently used as a crosslinker rather than conventional organic crosslinkers to form MXene-homopolymeric NC hydrogels. Zhang et al. have prepared $\mathrm{Ti}_{3} \mathrm{C}_{2} \mathrm{~T}_{x}$-polyacrylamide $\mathrm{NC}$ hydrogel using $\mathrm{Ti}_{3} \mathrm{C}_{2} \mathrm{~T}_{x}$ as a crosslinker. ${ }^{31}$ The aqueous suspensions of $\mathrm{Ti}_{3} \mathrm{C}_{2} \mathrm{~T}_{x}$ nanosheets were mixed with acrylamide under $\mathrm{N}_{2}$ atmosphere in the presence of potassium persulfate (KPS) to initiate the in situ polymerization of the acrylamide monomers. The crosslinking was attained by the hydrogen bonding between the $-\mathrm{CONH}_{2}$ groups of the polymer chains and the hydrophilic groups (-OH and $-\mathrm{F}$ ) of the $\mathrm{Ti}_{3} \mathrm{C}_{2} \mathrm{~T}_{x}$ nanosheets. When compared to a conventional organic crosslinked $N, N$-methylene bisacrylamide (BIS)-polyacrylamide hydrogel, the $\mathrm{Ti}_{3} \mathrm{C}_{2} \mathrm{~T}_{x}$-polyacrylamide hydrogel stands out with its lower crosslinking density and higher molecular weight of polymer chains.

\subsection{MXene-metal hybrid nanocomposite hydrogels}

To this point, the physically driven assembly of MXene nanosheets relying on self-gelation (Section 2.1) remains marginally explored with a need for optimized structural control. On the other hand, the use of GO and polymers as crosslinkers have demonstrated significant potential in realizing $3 \mathrm{D}$ interconnected MXene hydrogels with partial restacking of the nanosheets. Nonetheless, the inevitable oxidation during the previous gelation processes resulted in partial property degradation of the formed MXene hydrogels. Thus, to mitigate the 
oxidation effects, there is a need for a faster gelation process to accelerate the phase separation of MXenes from water and effectively suppress the restacking of the nanosheets. In this regard, Deng et al. promoted fast gelation of $\mathrm{Ti}_{3} \mathrm{C}_{2} \mathrm{~T}_{x}$ using divalent metallic ions, e.g., $\mathrm{Fe}^{2+}$, as crosslinkers to form a wellassembled MXene-metal hybrid hydrogel. ${ }^{17}$ The divalent ions were employed as the junctions interlinking the $\mathrm{Ti}_{3} \mathrm{C}_{2} \mathrm{~T}_{x}$ nanosheets relying on their strong interaction with the $-\mathrm{OH}$ surface groups (Fig. 6a). When added to a metal salt solution, i.e., $\mathrm{FeCl}_{2} \cdot 4 \mathrm{H}_{2} \mathrm{O}$, the strong bonding between $\mathrm{Fe}^{2+}$ and the $-\mathrm{OH}$ groups reduced the hydrophilicity of the negatively charged MXenes and facilitated their phase separation. Remarkably, the divalent metallic ion-initiated fast gelation (in few minutes) has efficiently prevented the oxidation of MXenes during the process.

To further validate the effect of metallic ions-diffusion on the gelation of MXene nanosheets, other multivalent metallic ions $\left(\mathrm{Mg}^{2+}, \mathrm{Co}^{2+}, \mathrm{Ni}^{2+}, \mathrm{K}^{+}\right.$, and $\left.\mathrm{Al}^{3+}\right)$ were also examined (Fig. 5b). It was shown that both divalent $\left(\mathrm{Mg}^{2+}, \mathrm{Co}^{2+}, \mathrm{Ni}^{2+}\right)$ and trivalent $\left(\mathrm{Al}^{3+}\right)$ ions were able to break up the electrostatic interaction between the MXene nanosheets and bond them together through the surface $-\mathrm{OH}$ groups. On the other hand, the added monovalent $\mathrm{K}^{+}$ions failed to initiate the gelation process and led to the coagulation of MXene nanosheets (Fig. 6b). This was attributed to the poor hydration energy of the monovalent ions compared to that of the divalent and
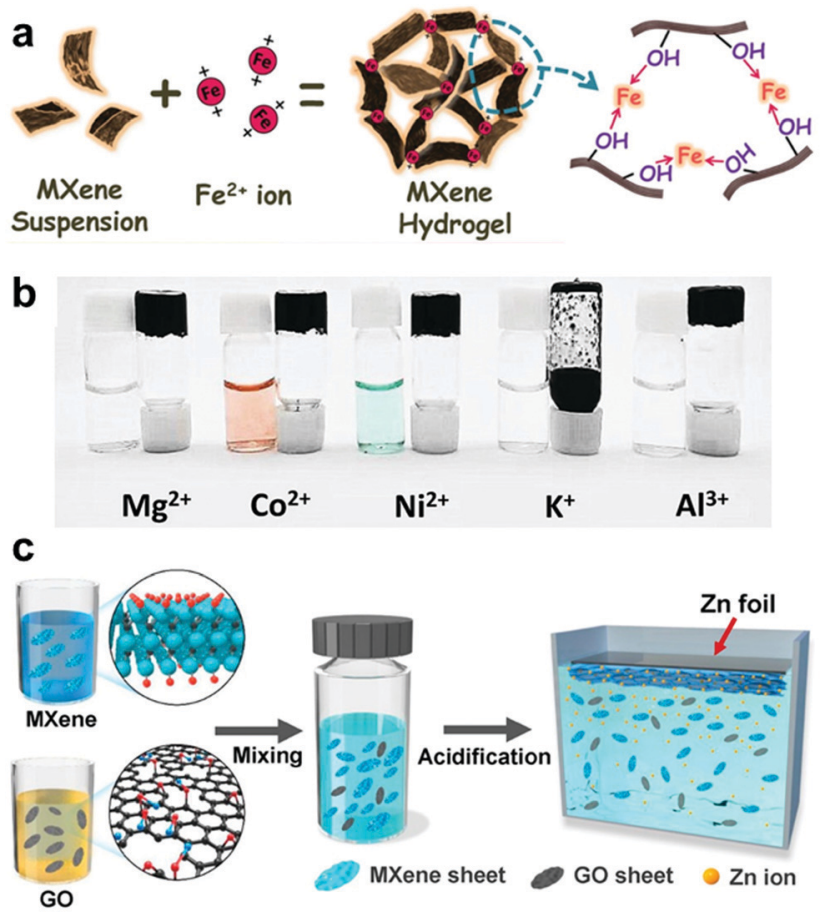

Fig. 6 (a) Schematic illustration of the $\mathrm{Fe}^{2+}$-initiated gelation process of MXene nanosheets and (b) effect of different multivalent metallic ions on the formation of MXene-metal hybrid hydrogels. Reproduced with permission from ref. 17 (copyright 2019, Wiley-VCH). (c) Schematic illustration of the $\mathrm{Zn}^{2+}$-mediated MXene-rGO NC hydrogels. Reproduced with permission from ref. 12 (copyright 2020, American Chemical Society). trivalent ions. However, the $\mathrm{Al}^{3+}$-assisted MXene hydrogel has shown a less developed 3D network with the presence of aggregated MXene nanosheets. The electrostatic forces between the MXene nanosheets were destroyed in a faster way by the extra positive charges associated with the trivalent ions. ${ }^{17}$

Recently, Lin et al. have simultaneously incorporated two crosslinkers, i.e., divalent metallic ions and GO, to prepare MXene-containing hydrogels through an improved gelation process. ${ }^{12}$ To initiate the gelation, a metal substrate, e.g., $\mathrm{Zn}$ foil, was placed on the surface of an acidified $\mathrm{Ti}_{3} \mathrm{C}_{2} \mathrm{~T}_{x} / \mathrm{GO}$ dispersion to release $\mathrm{Zn}^{2+}$ ions into the dispersion (Fig. 6c). The released $\mathrm{Zn}^{2+}$ ions participated in the co-existing MXeneinduced reduction of GO layers. With the diffusion of metallic ions $\left(\mathrm{Zn}^{2+}, \mathrm{Fe}^{2+}, \mathrm{Co}^{2+}, \mathrm{Ni}^{2+}\right.$, or $\left.\mathrm{Al}^{3+}\right)$, the negatively charged $\mathrm{Ti}_{3} \mathrm{C}_{2} \mathrm{~T}_{x}$ nanosheets were spontaneously assembled, forming a MXene-rGO hydrogel at the metal substrate. It is noteworthy that the divalent metallic ion-mediated gelation process, in the presence of a small amount of GO, has produced highly conductive MXene-rGO NC hydrogels with excellent structural robustness and stability against oxidation.

\subsection{Derivatives of MXene hydrogels: aerogels, xerogels, organohydrogels, and cryogels}

Owing to their distinctive properties, derivatives of MXene hydrogels are also of great interest for many applications. For instance, when compared to their hydrogel counterparts, MXene aerogels stand out for their lightweight, low density, high porosity, stable light-to-heat conversion efficiency, and large specific area. Such overwhelming merits make them promising candidates for microwave absorption, electromagnetic interference shielding, flexible electronics, and solar energy conversion. Thus far, there are several methods for preparing MXene aerogels including (1) direct freeze-drying of MXene NC dispersions, ${ }^{16,25,35,36}$ (2) calcination after freeze-drying of MXene NC dispersions, ${ }^{37-41}$ and (3) freeze-drying of MXene hydrogels. ${ }^{5,6,12,28,42-47}$ In all these methods, the sublimation of ice crystals from frozen dispersions or hydrogels was necessary for obtaining MXene aerogels. Similar to hydrogels, the resultant micro/nanostructures and mechanical behavior of the obtained aerogels are influenced by the MXene concentration, polymeric molecular weight, freezing methods, and post-processing procedure. A higher concentration of MXene nanosheets within a dispersion most likely would disturb interactions between polymers, resulting in decreased crystallinity. On the other hand, low polymeric molecular weight is less favorable due to the limited crosslinking sites on the surface of MXene nanosheets, which would lead to cracked porous structures. $^{25}$

Meanwhile, contrary to traditional freeze-drying which facilitates the formation of more disordered arrangement and structural collapse, directional freeze-drying was found effective in maintaining oriented lamellar micro/nano-structures. ${ }^{36}$ Besides, well-defined wave-shaped lamellae obtained by directional freeze-drying could serve as aligned "springs" to resist irreversible deformation under compression (Fig. 7a). For much lighter and well-assembled network, hollow MXene-rGO aerogel was obtained by exploiting polystyrene (PS) spheres as templates 


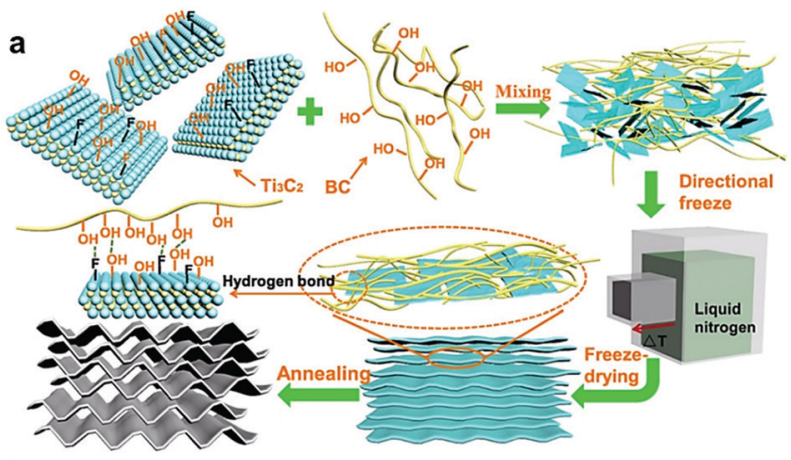

b

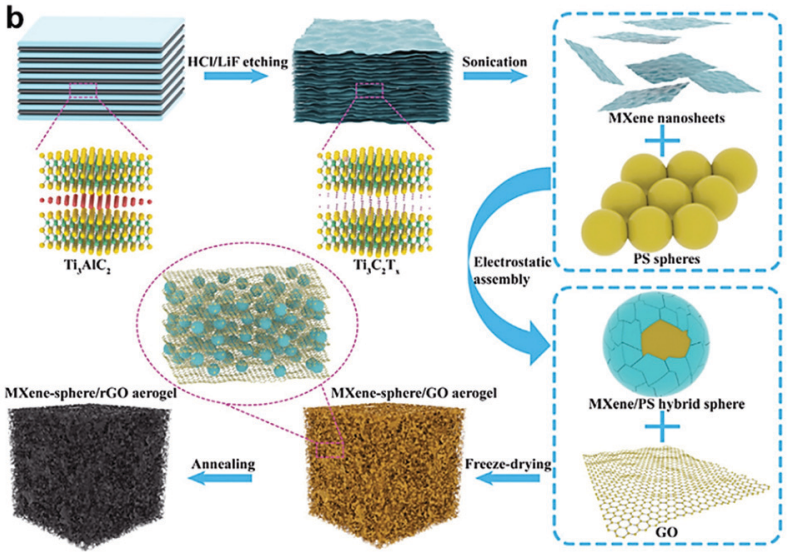

Fig. 7 Schematic illustrations showing the synthesis of MXene aerogels (a) MXene/bacterial cellulose carbon aerogel. Reproduced with permission from ref. 35 (copyright 2019, American Chemical Society). (b) Hollow MXene-rGO NC aerogels. Reproduced with permission from ref. 41 (copyright 2019, Wiley-VCH).

that were encapsulated by $\mathrm{Ti}_{3} \mathrm{C}_{2} \mathrm{~T}_{x}$ nanosheets and added to a $\mathrm{GO}$ solution (Fig. $7 \mathrm{~b}$ ). The $\mathrm{Ti}_{3} \mathrm{C}_{2} \mathrm{~T}_{x} / \mathrm{PS} / \mathrm{GO}$ mixture was freeze-dried and calcinated to remove the PS spherical templates, forming a freestanding 3D microstructured hollow $\mathrm{Ti}_{3} \mathrm{C}_{2} \mathrm{~T}_{x}-\mathrm{rGO}$ aerogels. During the compressing of the hollow MXene-rGO aerogel, multiple contact modes co-existed, such as point-to-point, point-to-sheet, and sheet-to-sheet, which in turn enhanced the sensitivity (Fig. 7b). The highly oriented-layered MXene aerogel skeletons obtained from freeze-drying of MXene hydrogels were found to improve the electromagnetic shielding ability by weakening the reflection of terahertz radiation. ${ }^{12}$ The subsequent calcination after freeze-drying was also beneficial for more gas generation and conduction within pure MXene-rGO aerogels, resulting in lower density and larger lamellar size. ${ }^{37}$

In addition to aerogels, MXene xerogels have also been reported. ${ }^{31,48}$ The main difference between the synthesis of aerogels and that of xerogels, is the way of extracting the liquid from the hydrogel. For aerogels, this process typically requires supercritical conditions to extract liquid, such as freeze-drying, while for xerogels the liquid can simply be evaporated at room temperature. Alternatively, aerogels can be turned into xerogels if subjected to thermal annealing at elevated temperatures, e.g., $500{ }^{\circ} \mathrm{C},{ }^{48}$ to collapse the $3 \mathrm{D}$ network of the aerogel. An example of the aerogel-xerogel transformation of MXene-PVA $\mathrm{NC}$ is presented in Fig. 8a. It follows the general pattern that a
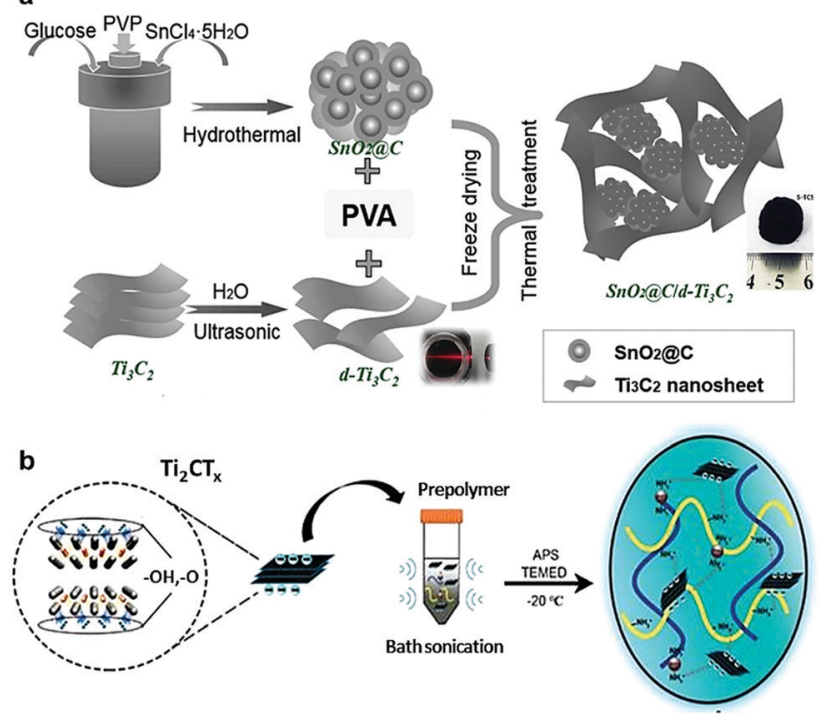

Fig. 8 Schematic illustrations showing the synthesis of MXene xerogels and cryogels. (a) Synthesis of 3D $\mathrm{SnO}_{2}\left(\mathrm{aC} / \mathrm{d}-\mathrm{Ti}_{3} \mathrm{C}_{2}-\mathrm{PVA}\right.$ xerogel. Reproduced with permission from ref. 48 (copyright 2018, Elsevier). (b) Fabrication of the $\mathrm{Ti}_{2} \mathrm{CT}_{x}$-cryogel through chemical crosslinking at $-20{ }^{\circ} \mathrm{C}$. Reproduced from ref. 21, under the terms of the Creative Commons License.

aerogels have a lower density than xerogels, and they retain their 3D structured network. On the other hand, xerogels easily shrink, get cracked, or even powdered. However, both types of gels are lighter than their parent hydrogels and offer a larger specific area.

Furthermore, the organohydrogel is another derivative of MXene hydrogels, which can be obtained by immersing a MXene-polymer NC hydrogel in a solution of an organic agent, e.g., ethylene glycol (EG), for a few hours to replace the water molecules. ${ }^{18}$ At first, the 'free' water molecules, which are not bound to the polymer chains, were rapidly exchanged with the EG molecules. While the intercalated water molecules, as well as the nonrotational bound water molecules, were slowly replaced by the EG molecules owing to the existing hydrogen bonding with polymer chains. The solvent displacement was effectively facilitated by the abundance of hydrogen bonds between EG and water, which prevented the evaporation of water molecules. Such a process offers an excellent solution for the considerable water loss challenge facing traditional hydrogels. Recently, one more derivative of MXene hydrogels, the cryogel, has been prepared using $\mathrm{Ti}_{2} \mathrm{CT}_{x}$ MXene rather than $\mathrm{Ti}_{3} \mathrm{C}_{2} \mathrm{~T}_{x}{ }^{21}$ Generally, the gelation of the precursors of a cryogel is achieved at subzero temperatures. In the case of MXene cryogel, the dried $\mathrm{Ti}_{2} \mathrm{CT}_{x}$ powder is added to a prepolymer solution during bath sonication, and then is kept overnight at $-20{ }^{\circ} \mathrm{C}$ (Fig. 8b), to allow for the chemical crosslinking process, i.e., cryogelation. It is worth mentioning that the formed cryogel has shown impressive stability at room temperature for more than 60 hours. Other conductive cryogels, prepared using GO, managed to maintain their stability for only 12 hours, indicating how MXene nanosheets in cryogels are closely interconnected. 


\section{Properties of MXene hydrogels and their derivatives}

MXenes have the general advantages of 2D materials including ultrathin structures, large specific surface area, and mechanical robustness. $^{7-10}$ However, MXenes possess several other properties that make them particularly attractive as reinforcements for hydrogels, providing a plethora of multi-responsive functionalities.

\subsection{Fundamental properties of MXenes}

Herein, we will touch on the key properties (Fig. 9a) of MXenes, particularly $\mathrm{Ti}_{3} \mathrm{C}_{2} \mathrm{~T}_{x}$, which probably is the most conductive
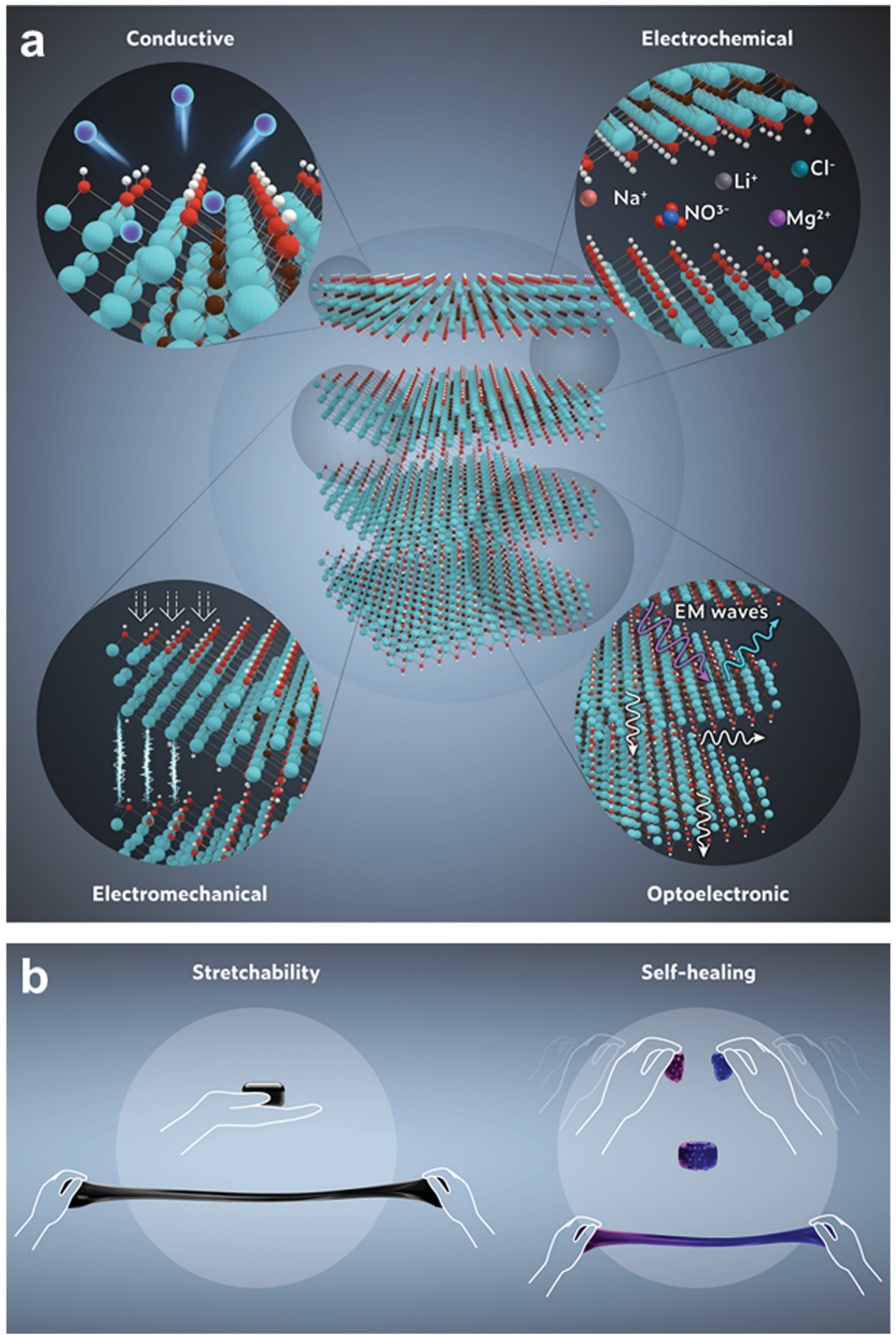

Fig. 9 Schematic representations of (a) the key fundamental properties of MXenes (e.g., $\mathrm{Ti}_{3} \mathrm{C}_{2} \mathrm{~T}_{x}$ ), and (b) the resultant exceptional stretchability and self-healing properties of MXene hydrogels. 
solution-processable 2D material, including graphene, making it appealing as an electrode material for electrochemical applications. ${ }^{7}$ In general, there are two kinds of electrode materials: (1) carbon-based materials, e.g., activated carbon, graphene, carbon nanotubes, carbide-derived carbon, laser scribed graphene, etc., which have high surface areas and good conductivity. (2) Oxides/hydroxides or transition metal dichalcogenides that have abundant active sites for ion intercalation or redox reactions. The formal kind has excellent power density, but low energy density. While the latter usually have a high energy density, but due to their lower electronic conductivity, they can not operate at high power density. In this regard, MXenes garner the advantages of both kinds of electrode materials. They have a conductive carbide core and a transition metal oxide/hydroxidelike surface with redox-active sites that can change their oxidation states during ion intercalation/exchange. It is worth mentioning that, unlike most of the $2 \mathrm{D}$ materials, the conductive core of MXenes is truly metallic with a high density of states at the Fermi level and high carrier concentrations, facilitating fast electron transport. $^{7,9}$ Such a combination indicates that further postsynthesis treatments (e.g., intercalation, ion exchange, and defect engineering), taking place at the surface of MXenes, will barely affect their electronic conductivity. They potentially attain high energy densities (like layered oxides) at much higher power densities (like carbon-based materials). This unique electronic structure of MXenes, especially $\mathrm{Ti}_{3} \mathrm{C}_{2} \mathrm{~T}_{x}$, has made them one of the greatest candidates for energy storage applications.

MXenes also have excellent mechanical properties. For instance, a $\mathrm{Ti}_{3} \mathrm{C}_{2} \mathrm{~T}_{x}$ monolayer was found to have the highest Young's modulus ( $c a$. $0.33 \mathrm{TPa}$ ) among all other solutionprocessable 2D materials, e.g., GO. ${ }^{10,14}$ Even when MXenes were combined with polymeric materials, e.g., PVA, the overall mechanical strength was further enhanced compared with pristine PVA. ${ }^{18}$ This highlights the potential of MXenes as gelators/cogelators to enhance the intrinsic mechanical properties of conventional polymeric materials. ${ }^{9,13,18}$ Furthermore, in line with its laminated structure, MXene nanosheets are known to have excellent compressive strength and relatively wide interlayer spacings, ${ }^{10,49}$ which allows for more flexibility and sensitivity under external forces. The combination of this high compressibility and the metallic conductivity gives rise to another electromechanical characteristic (Fig. 9a) that is unique to MXenes: whenever an external pressure is applied, the interlayer spacing will decrease, leading to a decrease in the internal resistance between the nanosheets and thus an increase in the conductivity, and vice versa. ${ }^{49}$ The combination of their outstanding mechanical and electrochemical properties, coupled with their large surface-to-volume ratio and excellent ion adsorption properties, makes MXenes attractive for stretchable and wearable electronics.

Furthermore, one of the most fundamental properties of MXenes is their excellent solution processability stemming from their inherent hydrophilicity. The negatively charged MXene surfaces associated with the abundant hydrophilic terminal moieties along with intercalated cations, increase the capacity of MXenes to interact with water molecules in a hydrogel network. ${ }^{8,9,14}$ With such rich surface chemistry, MXenes not only exhibit excellent electrical conductivity but also have a large number of free charge carriers, i.e., larger than that of other $2 \mathrm{D}$ materials, ${ }^{50}$ that can interact with the incident electromagnetic (EM) waves and reflect part of them before being absorbed. ${ }^{47}$ However, the local dipoles originating from the abundant surface functional groups were found to help with the absorption of the unreflected EM waves. Part of those absorbed incident waves will be transmitted through the whole multilayered stack of nanosheets, while the non-transmitted EM waves will be internally reflected by another nanosheet (Fig. 9a). This multifunctional ability to independently reflect, absorb, and/or transmit incident EM waves is indicative of the remarkable optoelectronic properties of MXenes. ${ }^{10}$ Owing to the abovementioned key properties when MXenes are used as crosslinkers/nanofillers for hydrogels, they introduce new functionalities to hydrogels and enhance their intrinsic mechanical properties, e.g., stretchability and self-healing capability (Fig. 9b). ${ }^{13,18}$ Thanks to their superior hydrophilicity, negative surface charge, rich stoichiometry, tunable surface functionality, and high-aspect-ratio nanostructures, MXenes can form hierarchically unoriented complex physical interconnections (e.g., chain entanglements, ionic interactions, and hydrogen bonding) with different polymers, leading to new gelation mechanisms of mechanically enhanced hydrogels. ${ }^{13,17,29,31,32,39}$ In return, MXenes also benefit from the inherent properties of the host gel network, which broaden their use in many applications requiring lightweight materials, swelling/de-swelling abilities, higher mechanical, and chemical stability. It is not an overstatement that the integration of MXenes into hydrogel systems has given rise to a genuinely unique hydrogel platform.

\subsection{MXene-induced new/enhanced properties in hydrogels}

When used as gelators, co-gelators, or nanofillers, MXenes could enhance the behavior of hydrogels in many aspects, and bring about exciting new functionalities. For example, from the rheological and electrochemical aspects, the behavior of the produced hydrogels was prominently affected by the characteristics of the parent MXene suspensions, e.g., concentration, size, and thickness of the nanosheets. For instance, dispersions made of either single or multi-stacked MXene nanosheets, at the same concentration, were found to have distinguishing elastic and viscous moduli, which would reflect on the rheological characteristics of the hydrogel. ${ }^{8}$ However, both suspensions exhibit almost the same shear-thinning behavior. ${ }^{10}$ Thus, for applications involving applied stresses, e.g., strain sensors, concentrated suspensions are often recommended to fabricate MXene hydrogels, regardless of the size of the nanosheets. Nonetheless, hydrogels made of thinner and larger MXene nanosheets (obtained by less intense etchants) have revealed a remarkable rheological tunability and better mechanical stability along with enhanced electrical conductivity. It was also found that hydrogel networks particularly made of thinner nanosheets exhibit better electrochemical performance than those with thicker multi-stacked nanosheets. ${ }^{22,23}$ However, for real-life applications, the performance of MXene hydrogels 
depends not only on its rheology but also on the collective behavior of its 3D structure. For example, when a combination of small and large MXene nanosheets was gelated together, forming an all- $\mathrm{Ti}_{3} \mathrm{C}_{2} \mathrm{~T}_{x}$ hydrogel, ${ }^{24}$ the attained electrochemical performance surpassed that of hydrogels incorporating nanosheets with close sizes. ${ }^{23}$ Such improved performance was ascribed to the combination of the high electronic conductivity of the large nanosheets and the high ionic conductivity of the small ones, as well as the increased interlayer spacing of the mixed sized MXene nanosheets.

Generally, in terms of electrochemical behavior, MXenebased gels are advantageous to their powder/film counterparts as well as other electrode materials (Section 4.1). This is attributed to the following: (1) the well-assembled 3D network prevents the restacking of MXene nanosheets, allowing for the exposure of more active surface sites, and better electrolyte permeability. (2) Metallic MXene networks provide highly continuous conductive pathways for efficient electron/ion transport. (3) The strong bonding of MXene with the electrolyte or other supporting materials in the gel matrix offers an effective electrode/electrolyte interface with low interfacial resistance. (4) MXene-containing gels possess an exceptional packing density higher than commercial activated carbon and graphite. ${ }^{21}$ Analogous to electrochemical behavior, the oxidation stability of the fabricated hydrogels is also affected by the dimensionality of the MXene nanosheets. ${ }^{8}$ Usually, hydrogels made of thinner and smaller nanosheets are more prone to oxidation, because of the higher ratio between the active surface area of the MXenes and their volume.

Tunable swelling capacity is another MXene-induced enhanced feature of their hydrogels, resulting from the negativelycharged hydrophilic surface of MXenes, and the uniform pore distribution within the hydrogel. With such swelling/ de-swelling abilities, 3D porous network, along with the abundant anchoring surface sites and polar terminal groups, several drugs can be easily grafted onto the MXene surfaces with higher uptake and release capacities. The remarkable biocompatibility attained by MXene-based hydrogels, i.e., higher than polymer hydrogels, is appealing for applications involving cell attachment and neuronal network growth. ${ }^{30}$

Apart from its biocompatibility, the 3D structure of the MXene gels was also found to lead to an improved EMI shielding efficiency (Section 4.4), because of its ability to create more scattering centers for the internally reflected EM waves, compared to interspaced MXene nanosheets (Fig. 9a). ${ }^{12,47}$

Finally, the inclusion of MXenes in hydrogel systems has patently improved the mechanical properties of the resultant hydrogel and even has brought about brand-new characteristics. In general, It is traditionally thought that the viscoelastic property of hydrogels is a disadvantage for mechanical sensing, as it can cause unstable baseline and fluctuation of signals if the stimulus is faster than the relaxation speed of hydrogels. However, in the case of MXene-based hydrogels, this characteristic viscoelasticity was shown to be an advantage for hydrogel-based sensors, particularly for sensing signals that involve complicated motions. ${ }^{13}$ Such an untraditional feature was attributed to the anisotropic mechanical responses of the MXene nanosheets within the gel matrix, which induce a much higher sensitivity towards compressive strains than that towards tensile ones. ${ }^{13}$

\subsection{Current issues and challenges}

Despite the remarkable capability of MXenes to be formulated into gels, they still have a metastable character, i.e., they are kinetically trapped materials. ${ }^{51}$ Therefore, the nanoscale structuring of such dynamic systems can change over time, particularly when the crosslinking of the gel networks is primarily associated with non-covalent physical interactions. Based on the collective behavior of each of the fabricated gels, the time-dependent structural changes of such dynamic systems would either lead to degradation or enhancement in the properties of the MXene-based gels. Thus, to properly engineer such morphological-dependent evolution of properties, several experimental parameters must be carefully controlled, especially given how variant is the gelation process of MXene gels. An example of these parameters is the CGC value of the incorporated gelators, which was only reported in detail in a few studies. ${ }^{29,30}$ Principally, the value of the CGC is vital for measuring the gelator efficiency and determining the compositions at which flowing gels, shake gels, or permanent hydrogels can be generated. ${ }^{4}$ Other significant parameters include heating and cooling rates, sonication conditions (if applied), size of the gel and the containing vessel, how the MXene gel is stored, and under what conditions, in addition to the age of the MXene-based gels when characterized. ${ }^{51}$ Following their gelation, an appropriate systematic characterization must be developed to assess the micro/macroscopic behavior of MXene-based gels. In this regard, several core characterization techniques have been applied, including scanning and transmission electron microscopy (SEM and TEM), different rheological methods, nuclear magnetic resonance (NMR), and Fourier transform infrared spectroscopy (FTIR). Indeed, employing every single technique is not a necessity. Instead, only specific combinations of techniques are required depending on the property-based design approach of the application-oriented reports on MXene-based gels. For instance, SEM and TEM are regularly used to obtain essential supramolecular-level information of all the MXene gels (e.g., morphology, porosity, and molecular arrangements), regardless of the purpose of the study. Rheological methods are also often used, especially for MXene hydrogels, to examine their flow properties and their behavior under mechanical stresses. NMR and FTIR are used to, respectively, probe the covalent and hydrogen bonding between the building blocks constituting the MXene gels. ${ }^{17,23,51}$ Well-designed fundamental characterization protocols are still needed to understand the structureproperty relationships governing the performance of this fast-growing hydrogel family. Understanding those relationships, which highly depend on the properties of the used gelators (e.g., MXene, rGO, polymers, etc.), chosen solvents (i.e., water in case of hydrogels), and gelation mechanisms, would help in expanding the application domains of MXene hydrogels and their derivatives. 
Another challenging factor is the precise control of the synthesis of MXenes and their corresponding hydrogels, which is particularly important for the practical application of MXene hydrogels and their derivatives. As elaborated in Section 2, the successful formulation of MXene hydrogels relies on the chemical/physical interactions between MXenes and other components in the gel matrix. However, full control over the gelation chemistry is essential for a precise selection of properties that can meet the demanding requirements of specific applications. Taking energy storage applications as an example, electronically conductive MXene hydrogels provide a continuous electron transport path because of their crosslinked chain; however, if the density of these chains is too high, less hierarchical pores will be created, which decreases ionic diffusion rates. A balance between electron and ion movement within the hydrogel is desired for optimized performances. Likewise, it is known that the interaction between polar functional groups in MXenes (e.g., $-\mathrm{F}$ and $-\mathrm{OH})$ and water molecules prompts its hydrophilicity; however, hydroxyls and fluorines are known to impede lithium-ion transport during the ion intercalation process. Hence, modifying the chemistries and synthesis protocols is crucial for the rational design of hydrogels for certain applications.

\section{Applications of MXene hydrogels and their derivatives}

As previously discussed, except for all-MXene hydrogels (Section 2.1), any other MXene gel, be it a hydrogel or any of its derivatives, combine the merits of both MXenes and the host gel matrix. Once the gelation is completed, the resultant behavior of the MXene-based hydrogels and their derivatives could either rely on the summation of the functionalities of the comprised components or stem from the synergistic interactions between those components, allowing for various potential applications. Fig. 10 summarizes the current application fields of MXene-based hydrogels and their derivatives.

\subsection{Energy storage}

4.1.1 Electrochemical capacitors (ECs). In principle, ECs store charges by the adsorption/desorption of electrolyte ions onto the surface of electrodes, and they can be categorized into electrostatic double-layer capacitors (EDLCs) and pseudocapacitors. The former stores the charges through pure electrostatic charge adsorption at the electrode/electrolyte interface, while the latter involves fast surface redox reactions or intercalationinduced redox reactions at the surface of the active materials.

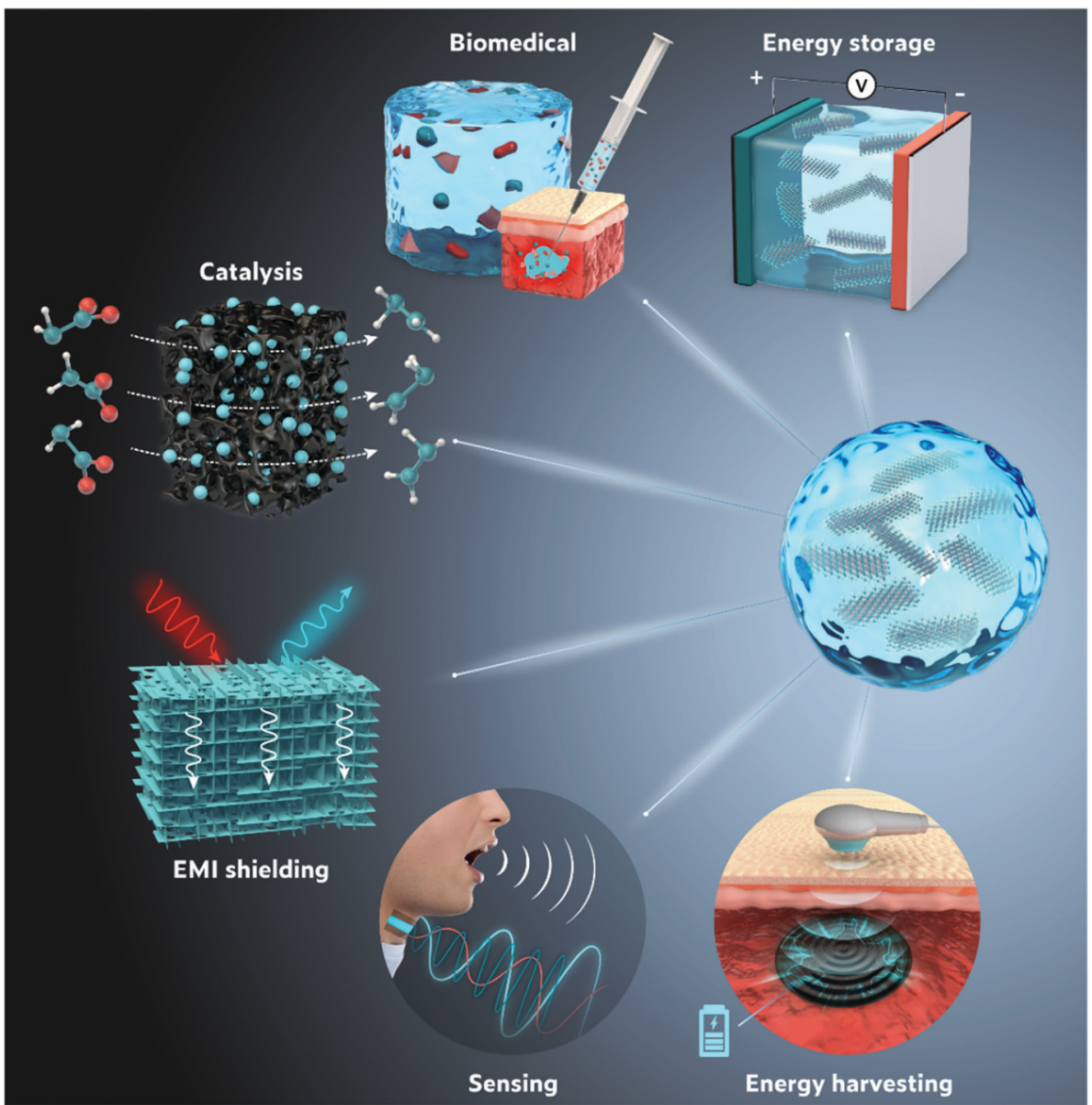

Fig. 10 Summary of possible applications involving MXene hydrogels and their derivatives. 
The electronic-transporting medium (electrode), ionic-transporting medium (electrolyte), and their interfaces, all play essential roles in the performance of a supercapacitor.

Thus far, different MXene-based hydrogels have been successfully utilized as electrodes for ECs, yet all of them were made of polymer-free networks. The first reported all-MXene hydrogel as a supercapacitor electrode was obtained by Lin et al., ${ }^{22}$ as discussed in Section 2.1. The corresponding all- $\mathrm{Ti}_{3} \mathrm{C}_{2} \mathrm{~T}_{x}$ hydrogel film had a capacitance of $70 \mathrm{~F} \mathrm{~g} \mathrm{~g}^{-1}$ with a wide potential window of $3 \mathrm{~V}$. However, when all-MXene hydrogel films were formed of delaminated nanosheets rather than the exfoliated ones, a high volumetric capacitance of $1500 \mathrm{~F} \mathrm{~cm}^{-3}$ was obtained while maintaining a gravimetric capacitance of $380 \mathrm{~F} \mathrm{~g}^{-1}$ (Fig. 11a), i.e., ca. $65 \%$ of its theoretical capacity $\left(615 \mathrm{C} \mathrm{g}^{-1}\right){ }^{23}$ This performance improvement was attributed to the high packing density as well as the inherent mechanical and electronic properties of the MXene. The $3 \mu \mathrm{m}$ thick $\mathrm{Ti}_{3} \mathrm{C}_{2} \mathrm{~T}_{x}$ hydrogel film showed a density of $c a .4 \mathrm{~g} \mathrm{~cm}^{-3}$, higher than that of commercial activated carbons $\left(c a .0 .5 \mathrm{~g} \mathrm{~cm}^{-3}\right)$ or even graphite ( $c a .2 .2 \mathrm{~g} \mathrm{~cm}^{-3}$ ), which renders a great potential for higher volumetric performance for real-world applications such as electric vehicles and miniaturized electronics. Such remarkable performance remained almost unchanged even when the electrode thickness was increased to $13 \mu \mathrm{m}$, with a volumetric capacitance of $1500 \mathrm{~F} \mathrm{~cm}^{-3}$ and a gravimetric capacitance of $370 \mathrm{~F} \mathrm{~g}^{-1}$ (Fig. 11b). The electrodes showed capacitance retention of more than $90 \%$ after 10k charge/discharge cycles at $10 \mathrm{~A} \mathrm{~g}^{-1}$ (Fig. 11c). The capacitive behavior and the low ion transport resistance were further confirmed using electrochemical impedance spectroscopy (EIS) as shown in Fig. 11d.

Following the work by Gogotsi and coworkers, ${ }^{23}$ Kayali et al. have further tuned the properties of the all-MXene hydrogel to realize different electrochemical and mechanical behaviors. They showed that by controlling the size of nanosheets, the trade-off between electronic and ion accessibility was optimized, which improved the electrochemical performance. ${ }^{24}$ Generally, larger MXene nanosheets have higher electrical conductivity because of the low contact resistance, while smaller ones offer much shorter ion diffusion pathways. Thus, when the hydrogel electrode was formed using a mixture of large (ca. $15 \mu \mathrm{m})$ and small (ca. 1-2 $\mu \mathrm{m})$ MXene nanosheets at a weight ratio of $1: 1$, a higher volumetric capacitance of $1513 \mathrm{~F} \mathrm{~cm}^{-3}\left(435 \mathrm{~F} \mathrm{~g}^{-1}\right)$ was achieved. ${ }^{24}$ The hydrogels retained $98 \%$ of their capacitance after $10 \mathrm{k}$ galvanostatic cycles. Remarkably, the capacitance achieved by the all- $\mathrm{Ti}_{3} \mathrm{C}_{2} \mathrm{~T}_{x}$ hydrogel was among the highest for MXene electrodes, demonstrating
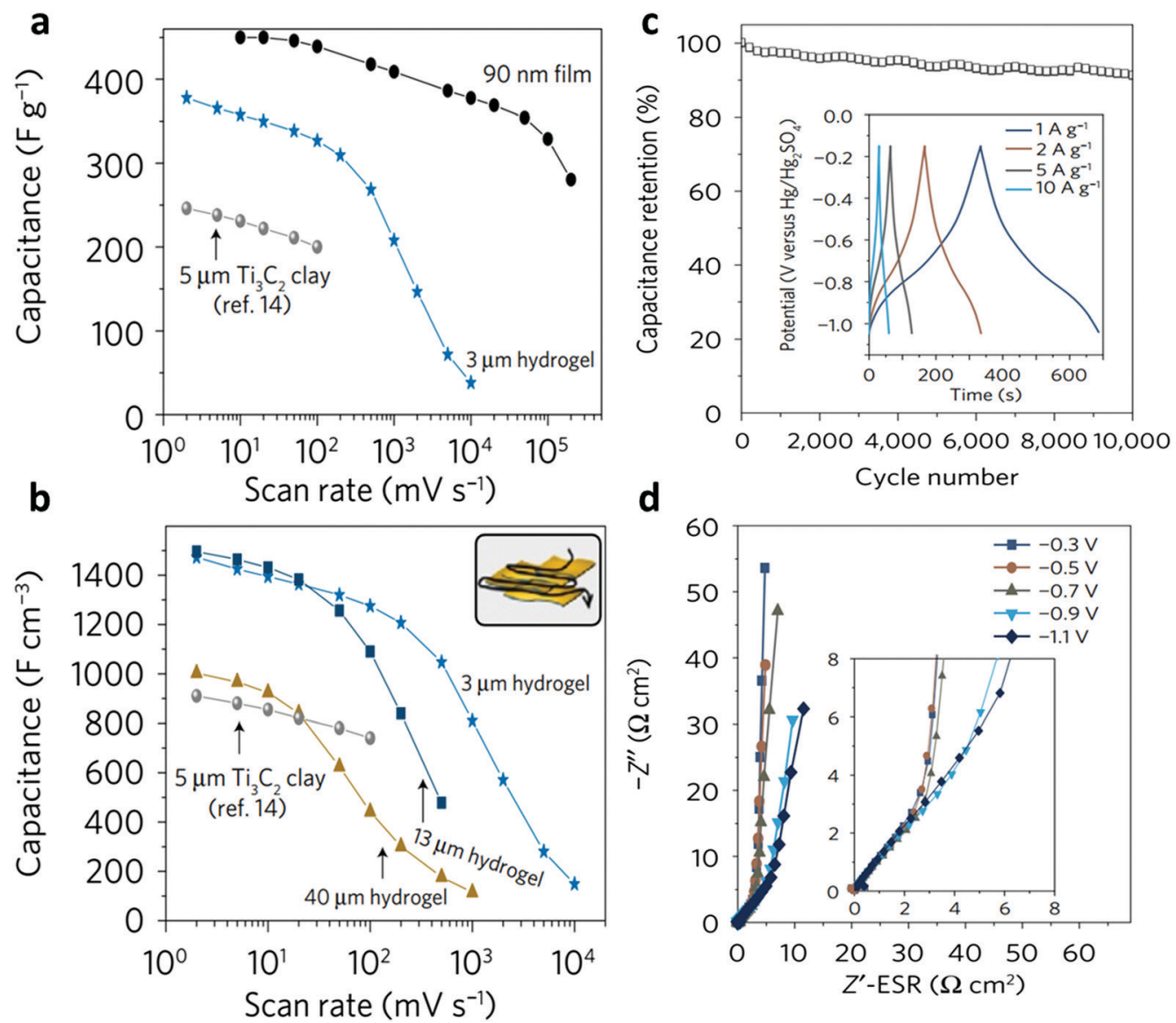

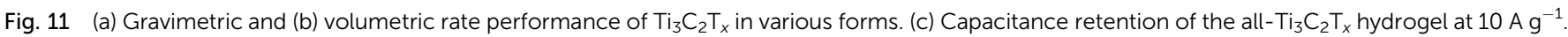

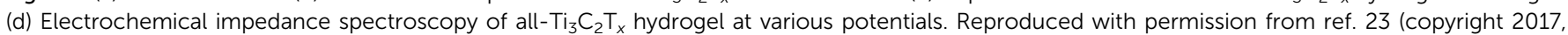
Nature Publishing Group). 
the great potential of MXene hydrogel-based electrodes in supercapacitors.

In another study, GO nanosheets were introduced as additional crosslinking agents in an attempt to improve the mechanical strength and flexibility of MXene hydrogel electrodes. Due to their $\pi$-conjugated system and abundant surface functional groups, GO provided good structural support for the MXene network. ${ }^{15}$ However, such structural stability was limited due to the relatively weak physical crosslinking between the $\mathrm{Ti}_{3} \mathrm{C}_{2} \mathrm{~T}_{x}$ and $\mathrm{rGO}$ nanosheets within the hydrogel network, ${ }^{26}$ leading to low electron transport efficiency at the interfaces when used for energy storage. To solve this problem, Shang et al. used an interlayer spacer (EDA) to chemically-bond the MXene and rGO nanosheets, as discussed in Section 2.2. ${ }^{29}$ The GOassisted hydrogel was mechanically robust and demonstrated excellent capacitance $\left(370 \mathrm{~F} \mathrm{~g}^{-1}\right.$ at $\left.5 \mathrm{~A} \mathrm{~g}^{-1}\right)$ and rate performance. Another approach to enhance the mechanical properties of the MXene hydrogels was attained by using divalent metal ions as interlinking joints between the MXene nanosheets, ${ }^{17}$ as explained in Section 2.4. The formed MXene-metal hybrid hydrogel exhibited a capacitance of $272 \mathrm{~F} \mathrm{~g}^{-1}$ at a scan rate of $2 \mathrm{mV} \mathrm{s}^{-1}$ and retained excellent rate performance $\left(226 \mathrm{~F} \mathrm{~g}^{-1}\right.$ at $1000 \mathrm{mV} \mathrm{s}^{-1}$ ).

4.1.2 Batteries. Beyond ECs, MXene NC hydrogels and their derivatives were also used in batteries. Generally, a battery is made of two active electrochemical electrodes separated by an ionically conductive separator. For either primary or rechargeable batteries, hydrogels bring more flexibility to rigid batteries, compared with conventional electrode materials, which is important to realize flexible/stretchable batteries for wearable electronics. Besides, the large interior space in the porous structure offers more active sites for the loading of active materials, without sacrificing the ionic or electronic conductivity. Although both aerogels and hydrogels share the advantage of the porous $3 \mathrm{D}$ network, water molecules are generally avoided to prevent the production of gas in the cell, since batteries are usually operated under high voltage (beyond the electrolysis voltage of water). Thus aerogels instead of hydrogels are more commonly used as electrodes for batteries. Besides electrodes, conductive hydrogels can also serve as binders for battery materials, owing to their ability to prevent the aggregation of oxide particles, and also promote ion and electron transport.

Although the direct application of MXene hydrogels as electrodes or binder materials for flexible batteries has not been reported yet, their derivatives (i.e., MXene aerogels) appear to be promising for loading various active battery materials, while maintaining good mechanical robustness. For example, freestanding MXene-rGO aerogels were shown to serve as lithium (Li) nucleation sites for Li-metal batteries. ${ }^{6}$ Due to the abundance of oxygen-functional groups, MXene-rGO aerogel was able to promote uniform plating/stripping of Li (Fig. 12a), while the crosslinked porous network provided good electron and iontransport paths (Fig. 12b). After compositional optimization, MXene-rGO aerogels could retain stable plating/stripping capacity up to $2.5 \mathrm{~mA} \mathrm{~h} \mathrm{~cm}{ }^{-2}$ (Fig. 12c). Fig. 12d and e show that this outstanding stability was maintained when the step-wise capacity increase from $1 \mathrm{~mA} \mathrm{~h} \mathrm{~cm} \mathrm{~cm}^{-2}$ to $5 \mathrm{~mA} \mathrm{~h} \mathrm{~cm}^{-2}$. Eventually, a full cell prototype with pre-plated GO-assisted MXene aerogel anode and $\mathrm{LiFePO}_{4}$ cathode was assembled, exhibiting a capacity of $149 \mathrm{~mA} \mathrm{~h} \mathrm{~g}^{-1}$ at $0.5 \mathrm{C}$ with good cycling performance (Fig. 12f). Furthermore, MXene-rGO aerogel was used as a polysulfide reservoir for lithium-sulfur batteries. ${ }^{45}$ The freestanding MXenerGO aerogel could provide a large space for accommodating the $\mathrm{Li}_{2} \mathrm{~S}$, and, due to the strong $\mathrm{Ti}-\mathrm{S}$ interaction, soluble polysulfide species could be efficiently adsorbed on the surface of MXene and promote the activation of $\mathrm{Li}_{2} \mathrm{~S}$ upon charging. As a result, high loadings of $\mathrm{Li}_{2} \mathrm{~S}$, up to $9 \mathrm{mg} \mathrm{cm}^{-2}$, was achieved at the MXenerGO cathode, along with a high areal capacity of $5.04 \mathrm{~mA} \mathrm{~h} \mathrm{~cm}^{-2}$ and good cycling stability.

\subsection{Biomedical applications}

Recently, MXene hydrogels have shown great promise for various in vivo biological applications, e.g., cancer therapy and drug release. Compared with hydrogels based on other 2D nanomaterials such as graphene, transition-metal dichalcogenides, and black phosphorus, MXene hydrogels offer the following advantages: (1) high hydrophilicity that promotes good dispersion and stability of MXene-derived photodynamic and photothermal agents in physiological media. (2) Anticancer drugs can be easily grafted onto MXene surfaces with the polar terminal groups. (3) Excellent anticancer drugs with loading capacities as high as $84 \%$ and high release percent, ${ }^{52}$ can be obtained by tuning the swelling performance of MXene hydrogels.

For instance, embedding anticancer agents into the MXenecellulose NC hydrogel was demonstrated to be an effective method for tumor therapy and controllable drug release. This was attributed to its multiscale polymeric 3D porous network, ensuring enormous anticancer drug (doxorubicin, DOX) uptake. ${ }^{30}$ Thanks to the $\mathrm{Ti}_{3} \mathrm{C}_{2} \mathrm{~T}_{x}$-induced high photothermal performance, killing cancer cells was achieved by allowing synergistic photothermal therapy (PTT) and chemotherapy (Fig. 13a). Under near-infrared (NIR) illumination at $808 \mathrm{~nm}$, the final lightinduced temperature of the $\mathrm{Ti}_{3} \mathrm{C}_{2} \mathrm{~T}_{x}$-cellulose/DOX NC hydrogel reached $55.3{ }^{\circ} \mathrm{C}$ at MXene concentration of 313.6 ppm. Remarkably, the high photothermal ability of MXene accelerated the drug (DOX) release. This was attributed to the vast volume expansion of the composite hydrogel under the NIR exposure, resulting in continual dynamic motions, during which the multiscale pores were enlarged to release the anticancer drug. Nearly $100 \%$ of the tumor cells were killed upon enhanced power intensity and irradiation durations with high light-toheat conversion (Fig. 13b-d). Compared to pure polymeric hydrogels, lower in vitro toxicity was demonstrated by MXenecellulose NC hydrogels, along with outstanding biocompatibility for four kinds of cells (HepA1-6, SMMC-7721, HepG2, U-118MG, and U-251MG).

Sanjiv et al. also reported a biocompatible sweet MXenechitosan NC hydrogel with a high swelling ratio, porous structure, and controlled degradation. ${ }^{34}$ The highly sweet $\mathrm{Ti}_{3} \mathrm{C}_{2} \mathrm{~T}_{x}$-chitosan NC hydrogel has expressed favorable biocompatibility towards 
a
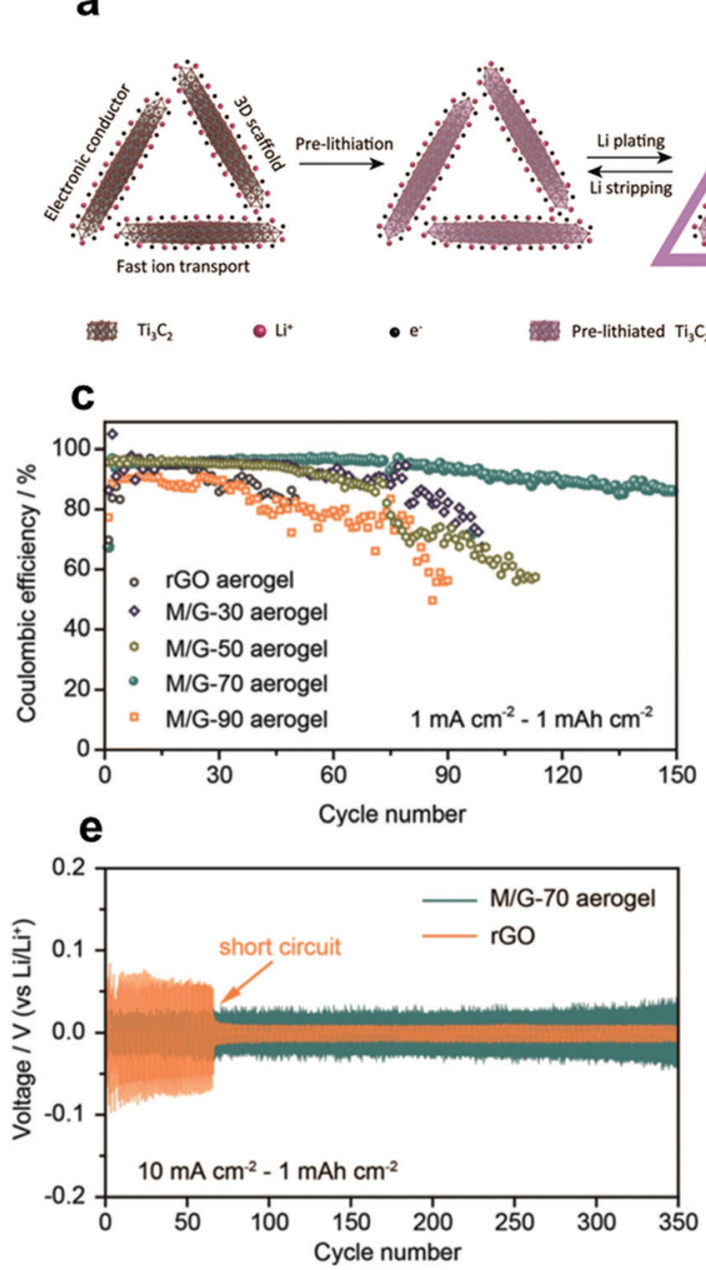

b

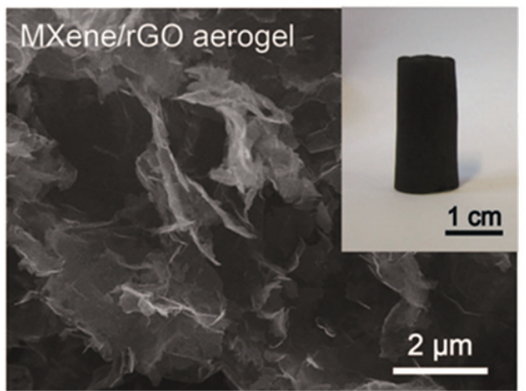

d
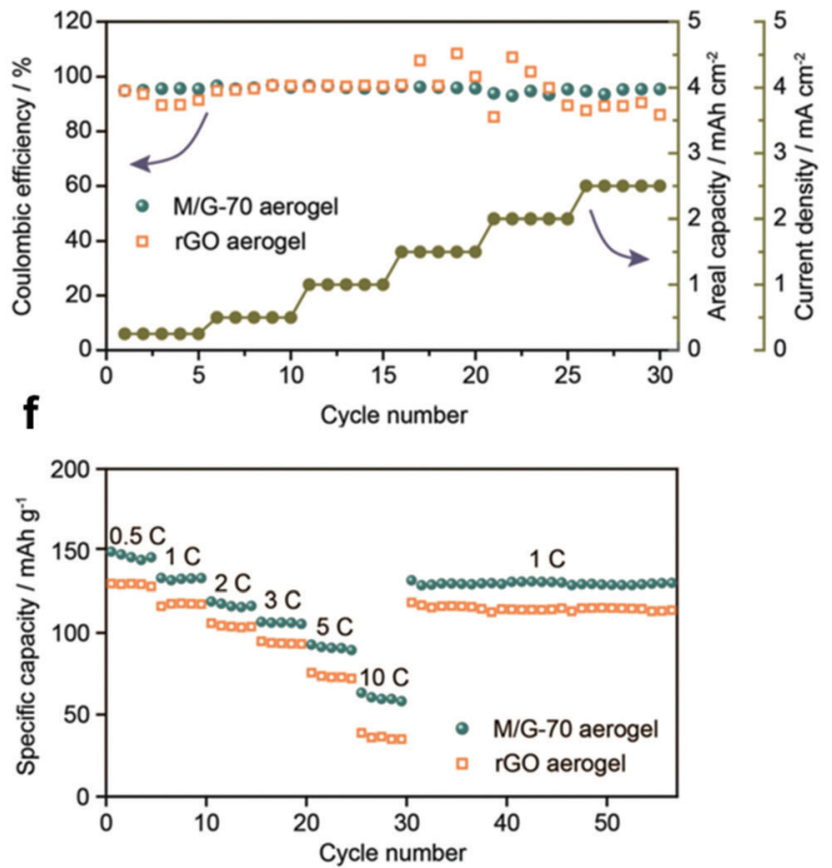

Fig. 12 (a) Fabrication of MXene-rGO aerogel electrode for lithium metal anode. (b) SEM image of the $\mathrm{Ti}_{3} \mathrm{C}_{2} \mathrm{~T}_{x}-\mathrm{rGO}$ aerogel. Inset: A digital photograph of the formed aerogel. (c) Coulombic efficiency of $r G O$ and different MXene-rGO aerogels at $1 \mathrm{~mA} \mathrm{~cm}^{-2}$. (d) Coulombic efficiency of the best MXenerGO aerogel (M/G-70) cycled at different current densities. (e) Voltage floating of the symmetric cells with M/G-70 aerogel scaffolds. (f) Cycling performance of the full cell with M/G-70 aerogel anode and LiFePO 4 cathodes. Reproduced with permission from ref. 6 (copyright 2018 , Wiley-VCH).

mesenchymal stem cells (MSCs) and cardiomyocytes over pure chitosan hydrogels, paving ways for their application in tissue regeneration. Furthermore, impressive drug-loading amounts (97.5-127.7 $\mathrm{mg} \mathrm{g}^{-1}$ ), sustainable release, and excellent release ratios (62.1-81.4\%) were demonstrated by MXene-polyacrylamide NC hydrogel with enhanced mechanical and swelling performances. ${ }^{31}$

\subsection{Catalysis}

Applications of MXenes have also been expanded to catalysis, and several MXenes have shown excellent catalytic activity towards different reactions. ${ }^{9}$ Besides, they are often used as catalyst supports because of their high conductivity, remarkable hydrophilicity, and rich surface chemistry. When they are formed into hydrogels, their performance as catalyst supports was further enhanced owing to the increase in the accessible active surface area. For instance, Chen et al. found that $\mathrm{Ti}_{3} \mathrm{C}_{2} \mathrm{~T}_{x}-\mathrm{rGO}$ hydrogels were able to sustain an interconnected porous network with open pores (Fig. 14a), whereas $\mathrm{Ti}_{3} \mathrm{C}_{2} \mathrm{~T}_{x}-\mathrm{rGO}$ powder has suffered from severe restacking (Fig. 14b). ${ }^{26}$ This structural difference consequently led to an increase in the Brunauer-Emmett-Teller (BET) surface area of the $\mathrm{Ti}_{3} \mathrm{C}_{2} \mathrm{~T}_{x}-\mathrm{rGO}$ hydrogel $\left(65 \mathrm{~m}^{2} \mathrm{~g}^{-1}\right)$, which is nearly two times as high as that of the mixed powder $\left(35 \mathrm{~m}^{2} \mathrm{~g}^{-1}\right)$ (Fig. 14c). By incorporating Eosin Y (EY) photosensitizer, the functional $\mathrm{Ti}_{3} \mathrm{C}_{2} \mathrm{~T}_{x}-\mathrm{rGO} / \mathrm{EY}$ NC hydrogel has exhibited an enhanced photoactivity, compared to its powder counterpart, toward both the reduction of $\mathrm{Cr}$ (vi) (Fig. 14d) and hydrogenation of 4-nitroaniline (Fig. 14e). For example, $99.3 \%$ of $\mathrm{Cr}(\mathrm{vI})$ was reduced in $10 \mathrm{~min}$ over the MXene-rGO/EY hydrogel under visible light illumination, while only $41.6 \%$ was converted over MXene-rGO/EY powder. This enhancement was attributed to the porous framework of hydrogel that increased the active surface sites and promotes the ion diffusion. A greatly increased transient photocurrent response of the $\mathrm{Ti}_{3} \mathrm{C}_{2} \mathrm{~T}_{x}-\mathrm{rGO} / \mathrm{EY}$ hydrogel was also observed (Fig. 14f), indicating that the separation of photogenerated charge carriers is more efficient over the MXene-rGO/EY hydrogel than that over the powder. Besides the improved catalytic activity, 


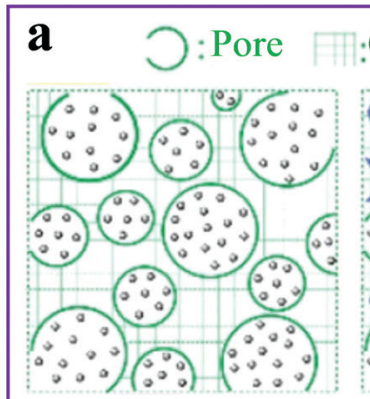

(a1)

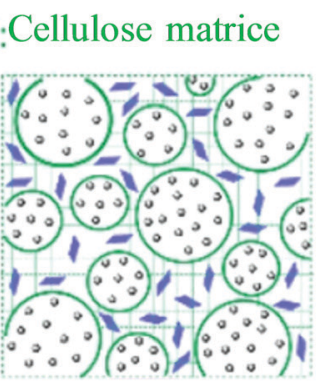

(a2)

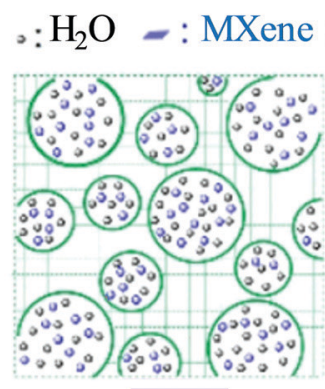

(a3)

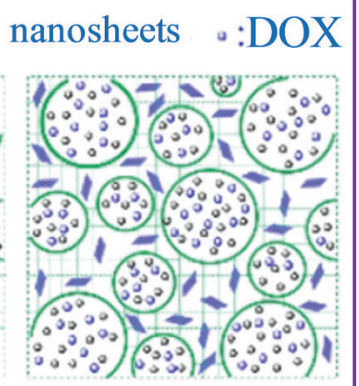

(a4)

\section{Cellulose Hydrogel Cellulose/MXene Cellulose/DOX Cellulose/MXene/DOX}

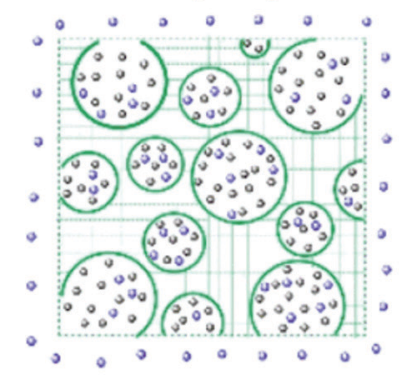

(a5)

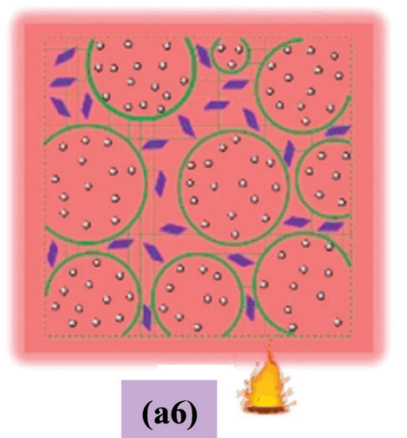

(a6)

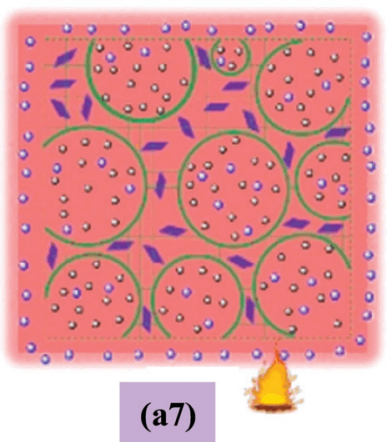

Bimodal PTT/Chemotherapy

Chemotherapy: DOX release

PTT

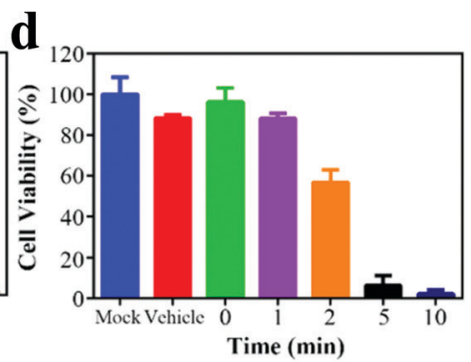

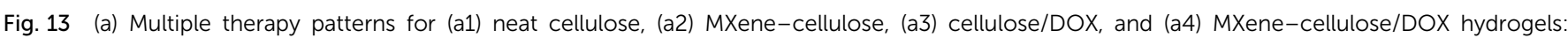

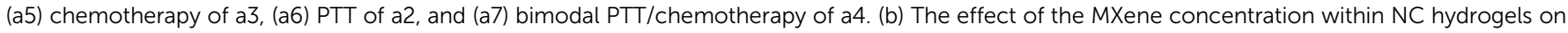

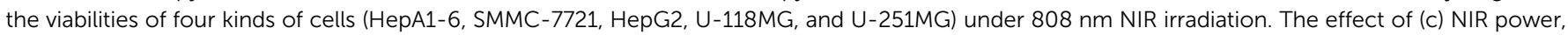

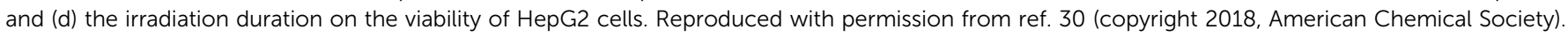

the $\mathrm{Ti}_{3} \mathrm{C}_{2} \mathrm{~T}_{x}-\mathrm{rGO} / \mathrm{EY}$ hydrogel has also shown an enhanced mechanical behavior and recyclability, allowing for its reuse without significant performance degradation.

The MXene-rGO hydrogels were also explored to support different noble metal catalysts. Yang et al. demonstrated that the Pt nanoclusters interact more strongly with $\mathrm{Ti}_{3} \mathrm{C}_{2} \mathrm{~T}_{x}$ than with graphene. ${ }^{28}$ They further developed a bottom-up approach to synthesize Pt-decorated $\mathrm{Ti}_{3} \mathrm{C}_{2} \mathrm{~T}_{x}-\mathrm{rGO}$ hydrogel and investigated its application in methanol oxidation. They found that $\mathrm{Ti}_{3} \mathrm{C}_{2} \mathrm{~T}_{x}$ can effectively tailor the electronic structures of $\mathrm{Pt}$ atoms and simultaneously offer numerous hydroxyl species. As a result, the $\mathrm{Pt}-\mathrm{Ti}_{3} \mathrm{C}_{2} \mathrm{~T}_{x}-\mathrm{rGO}$ hydrogel has exhibited high catalytic activity, good poison tolerance, and outstanding long-term stability. Similarly, Xie et al. explored the use of Pd-decorated $\mathrm{Ti}_{3} \mathrm{C}_{2} \mathrm{~T}_{x}$ hydrogel for catalytic hydrogenation of nitroaromatic compounds. ${ }^{27}$ The formed hydrogel was able to convert $91 \%$ of 4 -nitroaniline to $p$-phenylenediamine in $1 \mathrm{~min}$, in sharp contrast to $62.9 \%$ over $\mathrm{Pd}-\mathrm{Ti}_{3} \mathrm{C}_{2} \mathrm{~T}_{x}$ films. They further studied the catalytic activity of the $\mathrm{Pd}-\mathrm{Ti}_{3} \mathrm{C}_{2} \mathrm{~T}_{x}-\mathrm{rGO}$ towards the hydrogenation of other nitroaromatic compounds with different substituent groups. They found that the MXene-based hydrogel has shown higher activity for substrates with electron-donating groups (e.g. $-\mathrm{CH}_{3}$ and $\left.-\mathrm{OH}\right)$ than those with electron-withdrawing groups (e.g. $-\mathrm{Cl}$ and $-\mathrm{Br}$ ). The $3 \mathrm{D}$ network of the MXene $\mathrm{NC}$ hydrogel has effectively buffered the swelling behavior of $\mathrm{Ti}_{3} \mathrm{C}_{2} \mathrm{~T}_{x}$ during catalytic reactions, and therefore maintained a robust mechanical property. Though MXene hydrogels are considerably less explored as catalysts, their use as catalyst supports is expected to proliferate. Considering different metal catalysts, and the large family of MXene compounds (e.g., $\mathrm{Mo}_{2} \mathrm{TiC}_{2} \mathrm{~T}_{x}$ and $\mathrm{Nb}_{2} \mathrm{CT}_{x}$ ), many new MXene-based gel systems are expected to be developed for catalysis applications. 

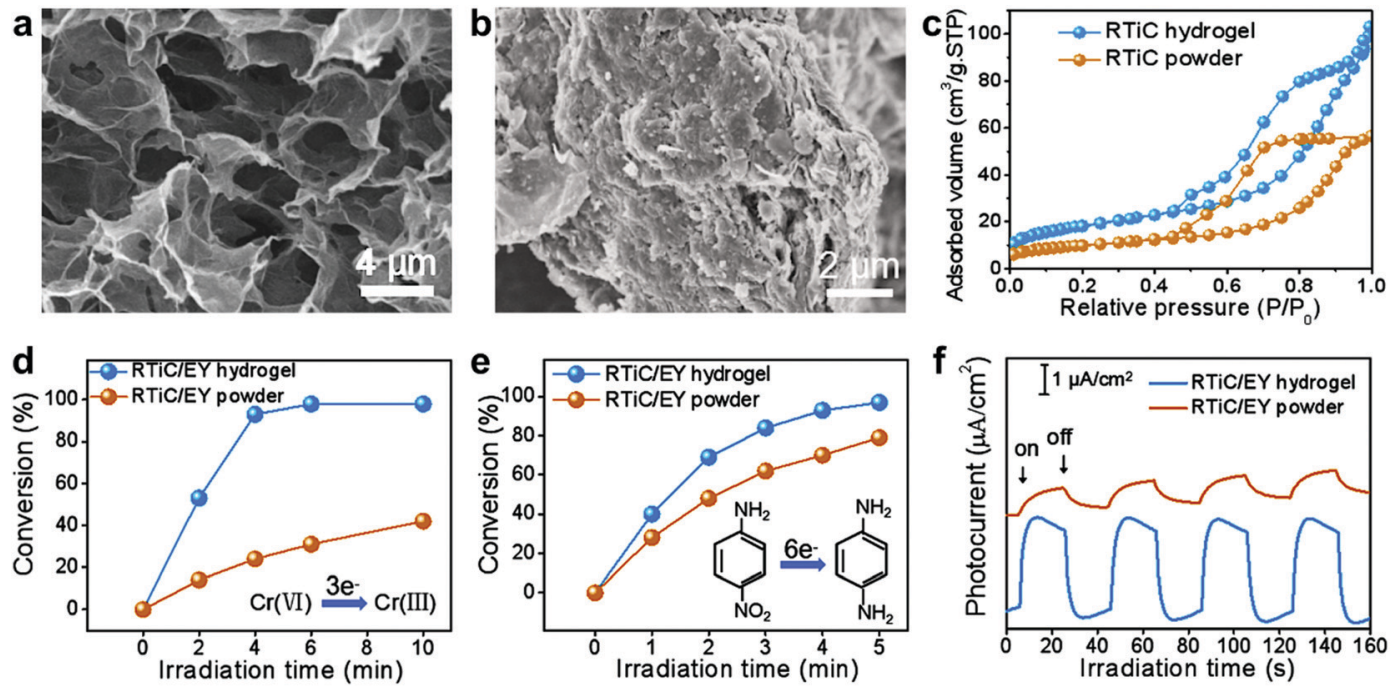

Fig. 14 SEM images of MXene-rGO (a) hydrogel and (b) powder. (c) $\mathrm{N}_{2}$ adsorption-desorption isotherms of $\mathrm{Ti}_{3} \mathrm{C}_{2} \mathrm{~T}_{x}-\mathrm{rGO}$ (RTiC) hydrogel and powder. The photocatalytic performance of (d) reduction of $\mathrm{Cr}(\mathrm{vI})$ and (e) hydrogenation of 4-nitroaniline under visible light irradiation. (f) Transient photocurrent spectra. Reproduced with permission from ref. 26 (copyright 2018, American Chemical Society).

\subsection{Electromagnetic interference shielding}

Nowadays, many technologies rely on massive amounts of connectivity for proliferating electronic nodes. These electronic devices keep dissipating a substantial amount of energy in the form of EMI, causing many electronic and electrical malfunctions. Hence, reducing the EMI by blocking the electromagnetic field with barriers made of conductive or magnetic materials can isolate the electronic device from the surroundings. Normally, the ability of barrier materials to block electromagnetic fields is evaluated by the EMI shielding effectiveness (SE), which is highly dependent on the conductivity of the material itself.

In this regard, many attempts have been made to increase the EMI SE of MXenes and extend their functionalities for potential applications. MXene-based gels with a 3D porous structure is a viable approach. Particularly, MXene hydrogels are commonly synthesized as the precursor for MXene aerogels for this specific application. Recently, when sodium alginate (SA) was employed as a spacer for MXene nanosheets, the obtained hierarchical structure was able to increase the scattering/reflection of the incident EM waves, and then the absorption of the transmitted EM waves. ${ }^{47}$ However, it was found that multiple reflections within the barrier structure have a significant impact on the EMI SE. In this regard, 3D porous MXene hydrogels stand out as ideal EMI shielding materials because they can create more scattering centers for the internally reflected EM waves, compared to interspaced nanosheets (Fig. 15a). Furthermore, when the MXene hydrogel is converted into a lightweight aerogel, higher conductivity is attained as a result of the removal of water molecules. For example, $\mathrm{Ti}_{3} \mathrm{C}_{2} \mathrm{~T}_{x^{-}}$ calcium alginate (CA) aerogel was reported to present excellent EMI SE of $54.3 \mathrm{~dB}$ at an ultrathin thickness of $26 \mu \mathrm{m}$, which is superior compared to commonly used carbon materials such as graphene and carbon nanotubes (Fig. 15b). To better compare the performance considering the density and thickness of the studied materials, specific shield effectiveness (SSE/ $t$, SE divided by sample density and thickness) was evaluated. The $\mathrm{Ti}_{3} \mathrm{C}_{2} \mathrm{~T}_{x}-\mathrm{CA}$ aerogel showed an outstanding SSE/t of $17586 \mathrm{~dB} \mathrm{~cm}^{2} \mathrm{~g}^{-1}$, which was higher than the $\mathrm{Ti}_{3} \mathrm{C}_{2} \mathrm{~T}_{x} / \mathrm{SA}$ film $\left(13195 \mathrm{~dB} \mathrm{~cm}^{2} \mathrm{~g}^{-1}\right)$ because of its unique porous structure, and therefore more reflection and scattering of EM waves (Fig. 15c).

Many other MXene aerogels were also proposed to enhance the mechanical properties of the MXene assemblies. For example, Zhao et al. developed a MXene-rGO aerogel with an aligned coreshell structure using GO-assisted hydrothermal method followed by freeze-drying. ${ }^{5}$ The MXene NC aerogel had an ordered cellular structure formed by rGO and compact $\mathrm{Ti}_{3} \mathrm{C}_{2} \mathrm{~T}_{x}$ nanosheets serving as a shell. High conductivity of $695.9 \mathrm{~S} \mathrm{~cm}^{-1}$ along with EMI SE of $56.4 \mathrm{~dB}$ was achieved at only 0.74 vol\% of MXene loading. Due to the good mechanical properties and large size of the rGO skeleton, the porous MXene NC aerogel has also demonstrated good mechanical strength, i.e., $100 \mathrm{mg}$ of MXene-rGO aerogel was able to support a load of $500 \mathrm{~g}$ when the force was applied along the direction of freeze-drying. Similarly, Fan et al. fabricated MXene-rGO aerogel, followed by annealing. The post-annealing process has led to partial removal of the surface functional groups of MXene. As a result, the NC aerogel exhibited higher electrical conductivity of $1000 \mathrm{~S} \mathrm{~cm}^{-1}$ and a high EMI SE of $50.7 \mathrm{~dB}$. It is worth noting that the MXene-rGO aerogels were not only active within the X-band frequency range, but it also had a good stealth property in the terahertz communication window. ${ }^{12}$

\subsection{Sensing}

Hydrogel sensors can respond to a wide range of chemical and physical stimuli by inducing measurable changes in geometric, optical, and electrical properties. Being a soft and stretchable sensing material has enabled their use in wearable/implantable electronics. However, when it comes to mechanical stimuli (strain/pressure), most hydrogel-based sensors suffer from low 

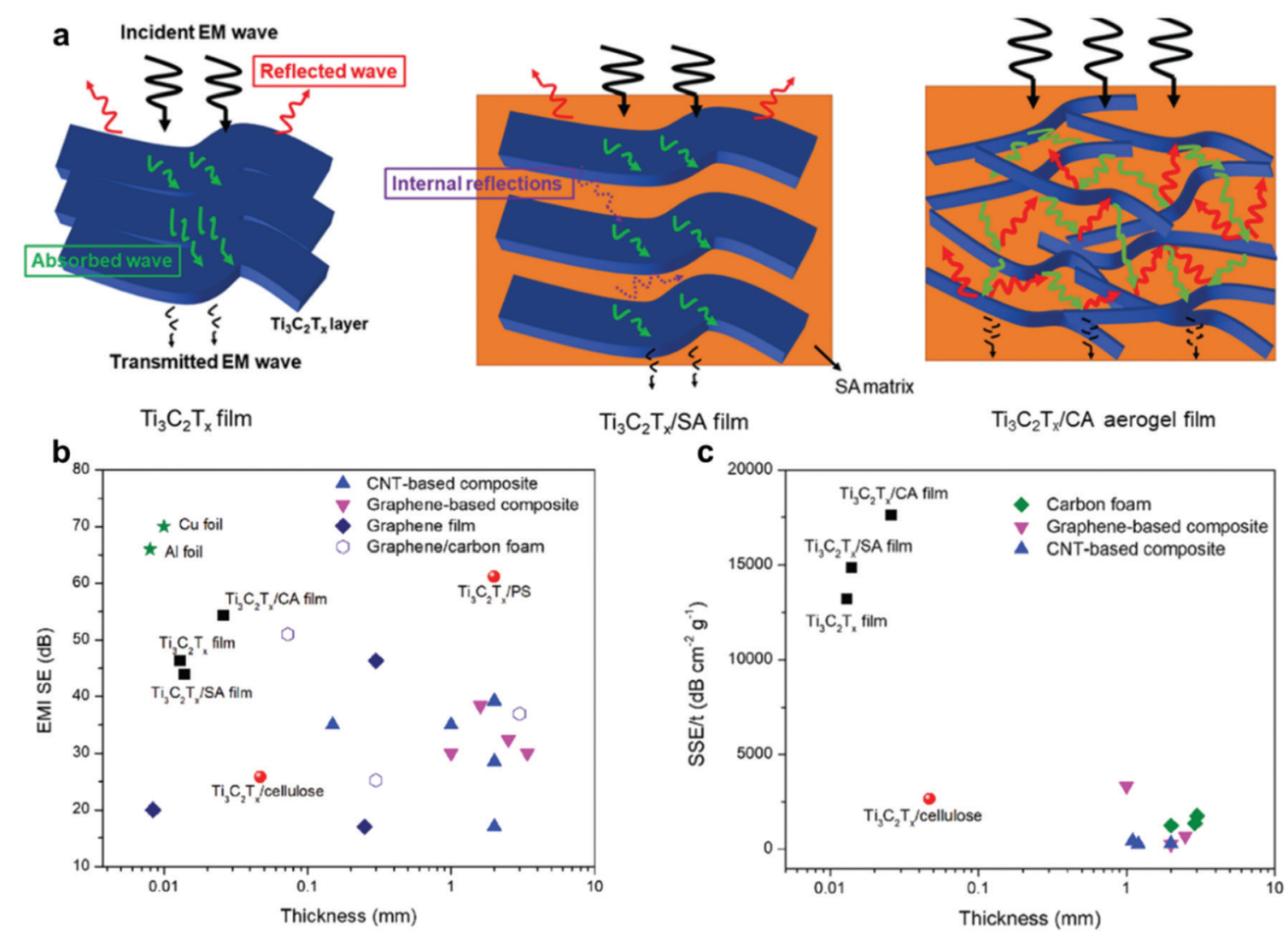

Fig. 15 (a) EMI shielding mechanism for freestanding $\mathrm{Ti}_{3} \mathrm{C}_{2} \mathrm{~T}_{x}$ film, $\mathrm{Ti}_{3} \mathrm{C}_{2} \mathrm{~T}_{x} / \mathrm{SA}$ film, and $\mathrm{Ti}_{3} \mathrm{C}_{2} \mathrm{~T}_{x}-\mathrm{CA}$ aerogel. (b) Thickness-dependent EMI SE of various materials. (c) Different SSE/t at different sample thicknesses. Reproduced with permission from ref. 47 (copyright 2019, Wiley-VCH).

sensitivity, and their electrical signals in response to external forces usually show hysteresis and fluctuation owing to their viscoelastic properties. To mitigate these challenges, we have developed a MXene-PVA hydrogel with a remarkable stretchability and self-healing ability. ${ }^{13}$ Interestingly, the hydrogel demonstrated high sensing capabilities towards different patterns of motions. The resistance of the MXene-based hydrogel increased (positive signal) when an object was moved on its surface parallel to the direction of the flowing current, whereas it decreased (negative signal) when the object was moving in the perpendicular direction. On the contrary, the resistance of the MXene-free PVA hydrogel was increased in both cases. Apart from motion direction, the MXene-PVA hydrogel was also able to sense the speed of motion when a cylinder was rolled down an inclined surface of the hydrogel. This interesting phenomenon can be understood in light of the deformation of the MXene-based hydrogel induced by the object moving at different velocities.

The abovementioned high sensitivity coupled with the exceptional sensing capability towards speed and directions of motions is appealing for certain advanced sensing applications. For instance, such a hydrogel can be used for the recognition of signatures which vary from one person to another in terms of force, speed, and writing sequence (Fig. 16a). Strikingly, the MXene-PVA hydrogel was able to distinguish between the 'OK' written by two different persons (Fig. 16b and c), whereas the pristine PVA hydrogel failed in recognizing the difference
(Fig. 16e and f). Not only that, but it was even possible to obtain a unique complex waveform for personalized signatures written on the $\mathrm{Ti}_{3} \mathrm{C}_{2} \mathrm{~T}_{x}-$ PVA hydrogel (Fig. 16d). ${ }^{13}$

Furthermore, the functionality of the phenomenal MXeneinduced sensitivity was extended by placing the MXene-PVA hydrogel on the "Adam's apple" (Fig. 17a) of a speaking person. Interestingly, the MXene-based hydrogel could provide orders of magnitude richer information about the phonatory (voicing) process (Fig. 17b-d) than in the case of pristine PVA hydrogel (Fig. 17e-g), and thus facilitated the differentiation of similarly sounding syllables of "B", "D" and "E". These findings highlight the advantageous viscoelastic characteristic of $\mathrm{Ti}_{3} \mathrm{C}_{2} \mathrm{~T}_{x}$-PVA hydrogels, thanks to the MXene-induced anisotropic responses to compressive and tensile strains, particularly for sensing signals that involve complicated motions such as touch and various physiological ones.

The versatility of the MXene-based hydrogel sensors was further extended to MXene-PAAm-PVA organohydrogels (refer to Sections 2.3 and 2.5 for synthesis details). ${ }^{18}$ The abundant hydrogen bonding between water and the organic (ethylene glycol) molecules was able to both disrupt the formation of crystal lattices of ice and hinder water evaporation, leading to anti-freezing and water-retaining properties, respectively. The excellent self-healing ability can be attributed to the dynamic crosslinking and supramolecular interactions between the various components of the organohydrogel. ${ }^{53,54}$ As a result, 

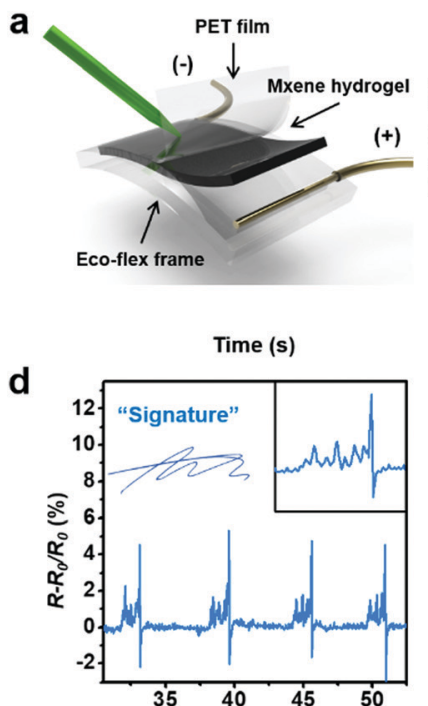
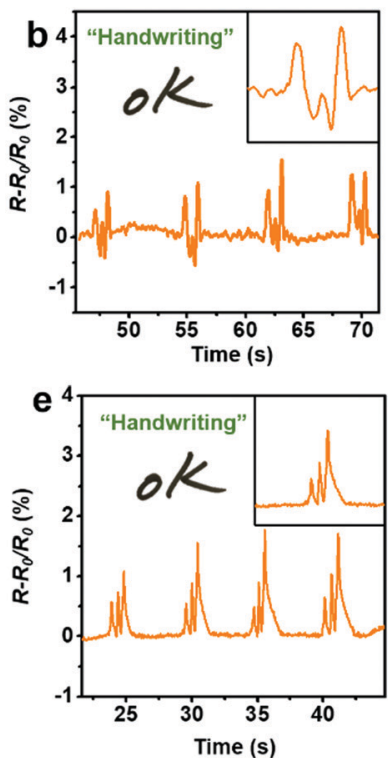
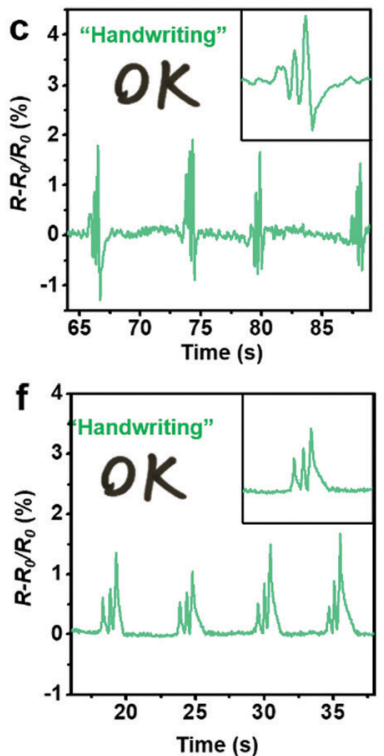

Fig. 16 (a) Schematic for handwriting sensing. ( $b$ and c) Resistance change of the MXene-PVA hydrogel to the word 'OK' that is handwritten by two different volunteers, and (d) a handwritten signature. (e and f) Resistance change of pristine PVA hydrogel towards 'OK', written by the same two volunteers. Reproduced with permission from ref. 13, copyright 2018, American Association for the Advancement of Science.
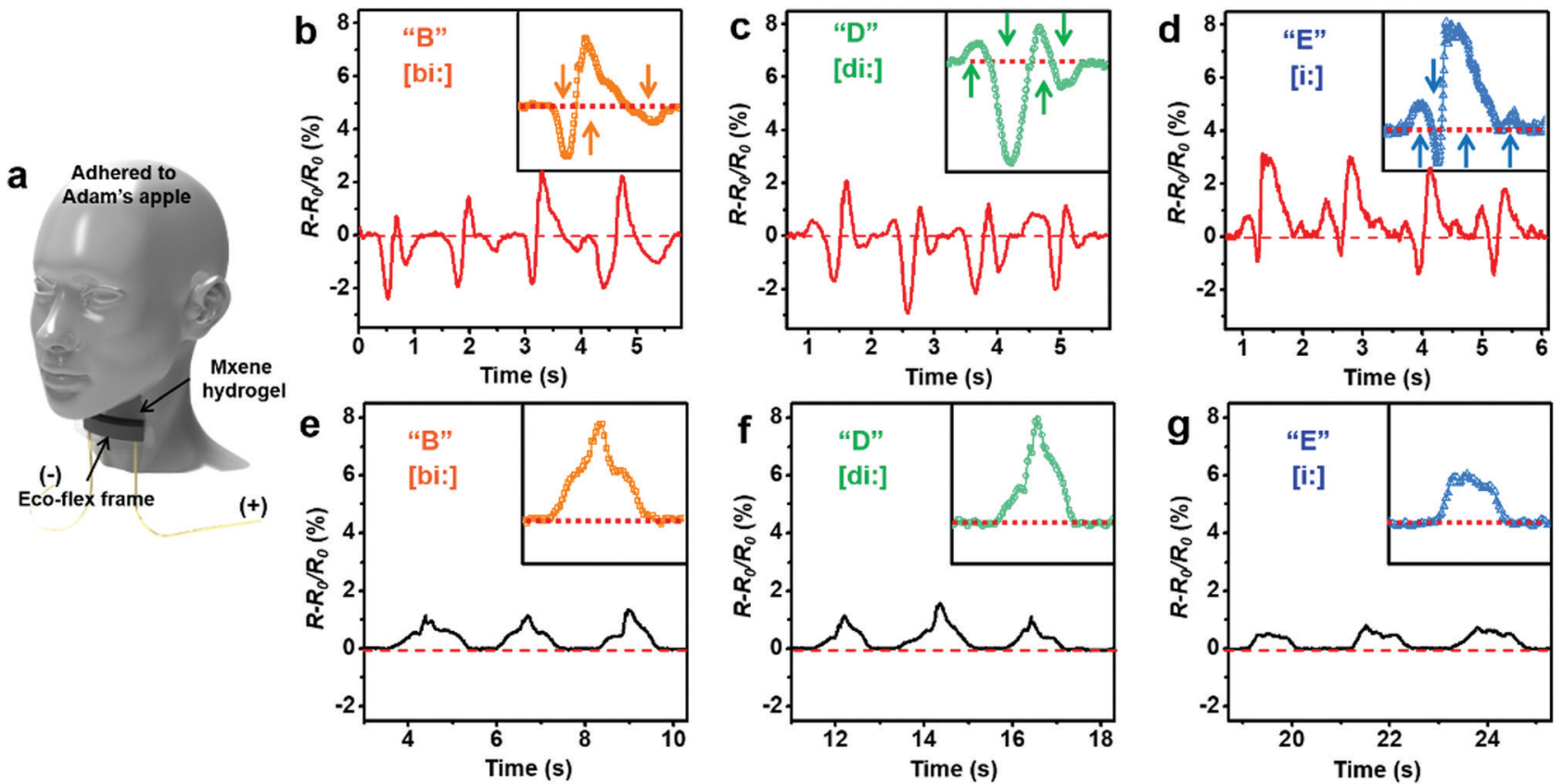

Fig. 17 (a) Schematic for vocal sensing, showing the MXene-PVA hydrogel, with an ecoflex frame, adhered to Adam's apple of a speaking person. $(b-d)$ Resistance change of the MXene-PVA hydrogel in response to similarly sounding letters " $B$ ", " $D$ ", and "E". (e-g) Resistance change of pristine PVA hydrogel in response to "B", "D", and "E". Reproduced with permission from ref. 13, copyright 2018, American Association for the Advancement of Science.

the MXene-copolymeric organohydrogel was used as a wearable strain sensor to detect various human activities such as finger bending and swallowing with wide strain range (up to $350 \%$ strain) and high sensitivity even at temperatures as low as $-40{ }^{\circ} \mathrm{C}$.

Another MXene-based hydrogel derivative (aerogel) was used as a compressible conductive material for sensing applications. For example, Gao et al. fabricated a wearable pressure sensor made of MXene-rGO aerogels. ${ }^{39}$ The 3D porous network of the aerogel has allowed for sensing a wider pressure range with higher sensitivity than that of MXene-rGO filtrated films. Besides, the strong interaction between MXene and rGO nanosheets has led to improved mechanical properties compared to pure rGO aerogels. The optimal MXene-rGO aerogel has exhibited impressive stability over 10000 sensing cycles, 
with a sensitivity as high as $22.56 \mathrm{kPa}^{-1}$. Such sensitivity can enable the detection of tiny yet vital signals relevant to human health, such as pulses under peaceful conditions.

\subsection{Energy harvesting}

When it comes to implantable medical devices such as pacemakers and neurostimulators, it is necessary to ensure their smooth operation for long time (preferably dozens of years), which requires energy sources with unlimited capacity. Among the potential energy sources, ultrasound waves stand out for their easy accessibility and low attenuation through human tissues, allowing for harmless charging of implantable devices. However, most of the available implantable energy harvesting devices require specific materials and complicated fabrication processes, and they often lack proper biocompatibility.

In this regard, by using the same MXene-homopolymeric hydrogel developed for sensing, ${ }^{13}$ we have demonstrated that MXene hydrogels can be used for harvesting ultrasound energy. ${ }^{19}$ Fig. 18a depicts an extremely simple device structure consisting of a layer of $\mathrm{Ti}_{3} \mathrm{C}_{2} \mathrm{~T}_{x}$-PVA hydrogel sandwiched between two ecoflex covers. When the ultrasound tip was put in direct contact with the MXene-based device, the output voltage reached values as high as $2.8 \mathrm{~V}$ (Fig. 18b). At a given frequency $(20 \mathrm{kHz})$, the voltage varied with the incoming ultrasound wave (Fig. 18c). Moreover, the device was able to operate in mediums similar to biological tissues (water, hydrogel, and ecoflex) at different spacings (medium thickness) a
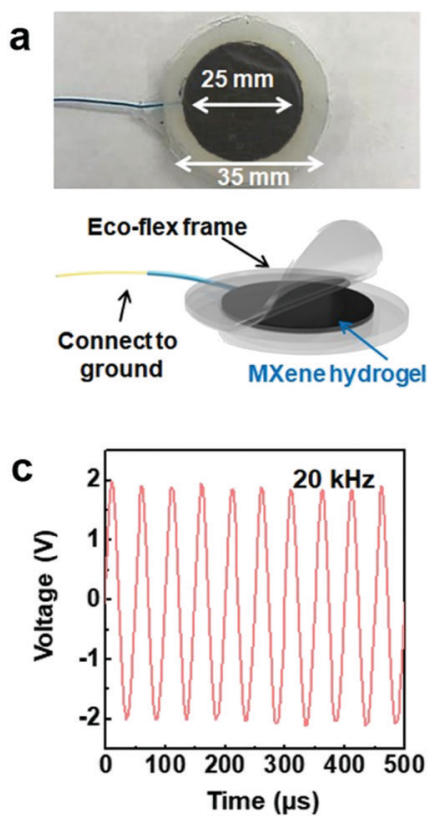

b
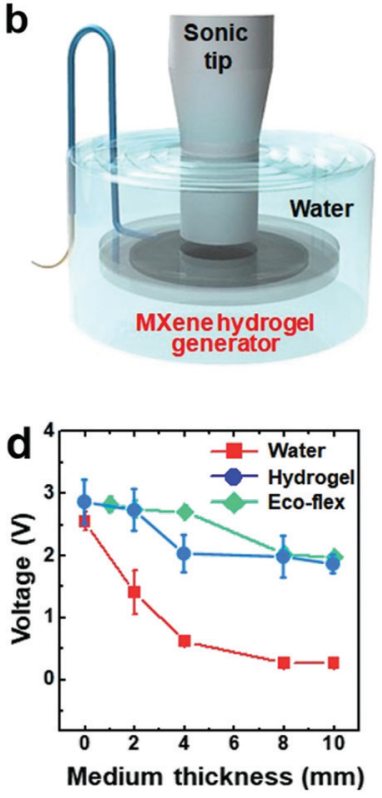

Fig. 18 (a) Photo and schematic illustration of the MXene-PVA hydrogel generator. (b) Schematic showing the testing setup used to study the MXene-based generator. (c) The voltage waveform produced by the $\mathrm{Ti}_{3} \mathrm{C}_{2} \mathrm{~T}_{x}$-PVA hydrogel generator, at $20 \mathrm{kHz}$. (d) Output voltages of the $\mathrm{Ti}_{3} \mathrm{C}_{2} \mathrm{~T}_{x}$-PVA generators in water, ecoflex, and hydrogel mediums, as a function of the medium thickness. Reproduced with permission from ref. 19 (copyright 2020, American Chemical Society). between the tip and the device (Fig. 18d), showing its potential for in vivo charging application.

The interesting operation mechanism of the $\mathrm{Ti}_{3} \mathrm{C}_{2} \mathrm{~T}_{x}-\mathrm{PVA}$ hydrogel energy harvester was explained using the so-called streaming current/voltage theory. In the absence of the external field, an electric double layer (EDL) can be formed on the negatively charged MXene surfaces (Fig. 19a). When the ultrasound is on, the pressure-driven flow can push/drag excess counter ions within the EDL towards/away from the MXene surfaces, generating an oscillating voltage/current that fits the pattern of the incoming ultrasound waveform (Fig. 19b). This explanation was corroborated based on the fact that the output voltage was highly dependent on the ionic state within the hydrogel, which was illustrated by adding different amount of $\mathrm{H}_{2} \mathrm{SO}_{4}$ into the hydrogel system (Fig. 19c).

Although the triboelectric effect was not sufficient to explain the power generation phenomenon, it was utilized to improve the performance of the MXene-PVA hydrogel generator. By simply introducing a tribo-pair, i.e., placing a layer of nylon between the hydrogel and the ecoflex (Fig. 20a), a triboelectric charge induction took place, causing an increase in the charge density at the EDL. This has led to a significant increase (four times) in the output voltage, reaching $8 \mathrm{~V}$ at a load resistance of $10 \mathrm{M} \Omega$ (Fig. 20b). By measuring the output voltage and current of the MXene-based NC hydrogel generator as a function of the load resistance (Fig. 20c), the optimal load resistance was determined to be $c a .100 \mathrm{k} \Omega$. This has led to a maximum power of $21.8 \mu \mathrm{W}$, with a power conversion efficiency of $0.021 \%$, which is comparable to that of the state-of-the-art triboelectric generators (Fig. 20d). ${ }^{55}$ With such a high output power, MXene-based hydrogel generators can operate common electric gadgets, e.g., electric hydrometers. Interestingly, when
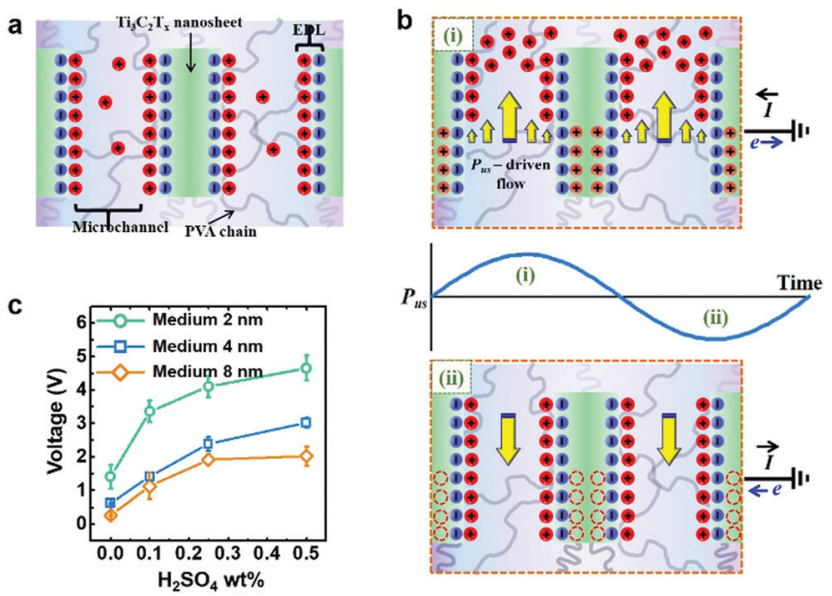

Fig. 19 The proposed mechanism for voltage generation phenomenon in the ultrasound-driven MXene-homopolymeric hydrogel generators. (a) Schematic showing the formation of the electrical double layer (EDL) within the $\mathrm{Ti}_{3} \mathrm{C}_{2} \mathrm{~T}_{x}$-PVA hydrogel. (b) Schematic illustration of the proposed electrical signal generation mechanism of MXene-PVA driven by the propagation of the ultrasound wave. (c) Output voltage profile of the MXene-PVA hydrogel generators as a function of $\mathrm{H}_{2} \mathrm{SO}_{4}$ wt\% in the hydrogel using an ecoflex medium of various thicknesses. Reproduced with permission from ref. 19 (copyright 2020, American Chemical Society). 
a
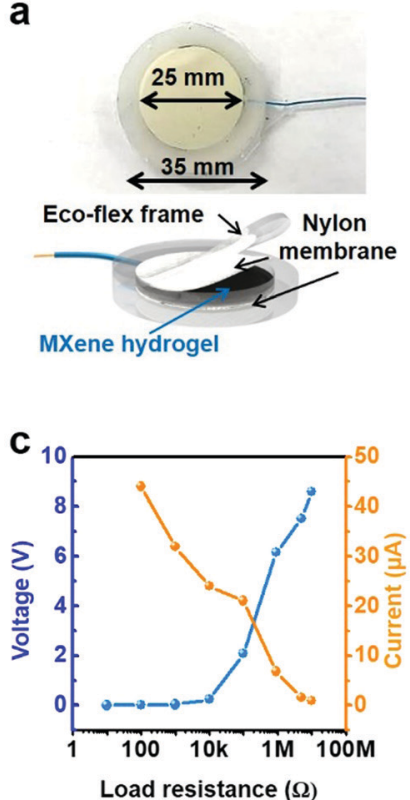

b
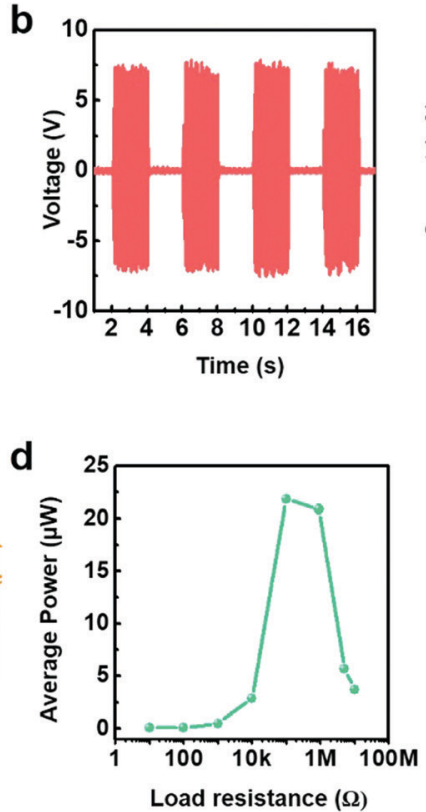

e

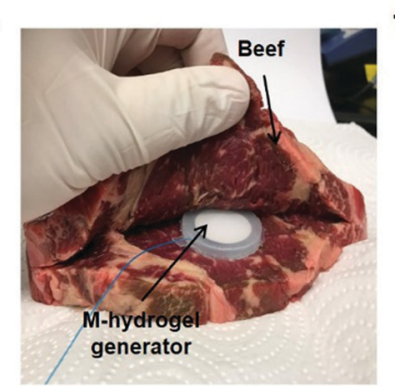

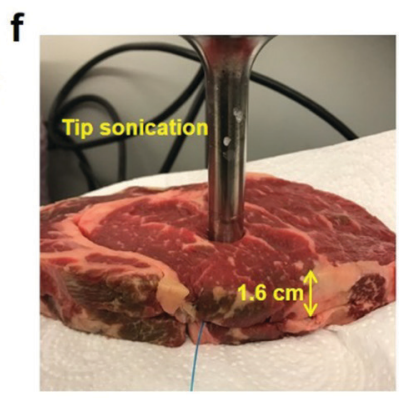

g

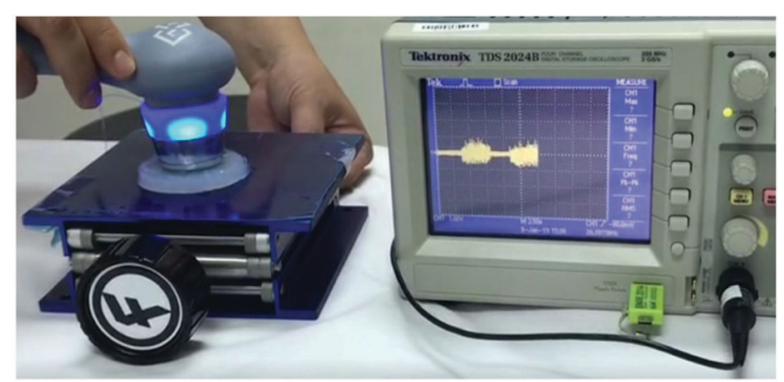

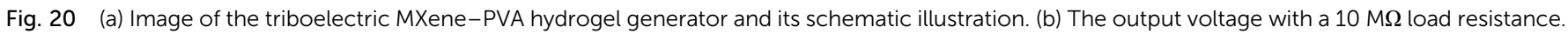

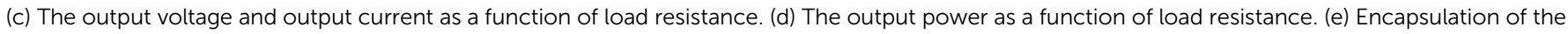

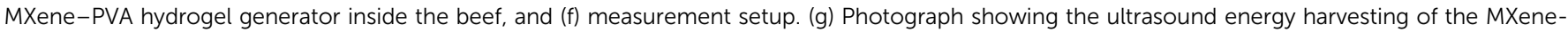

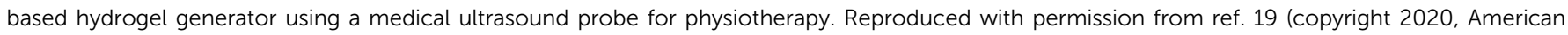
Chemical Society).

the generator was buried within a slice of fresh beef, it was still able to produce a voltage of $4.5 \mathrm{~V}$, which highlights its feasibility for in vivo applications (Fig. 20e and f). It is worth mentioning that MXene-based hydrogel generators were also able to harvest ultrasound energy from common medical ultrasound probes for both imaging and physical therapy (Fig. 20g).

\section{Conclusions and perspectives}

In this tutorial review, we have discussed in detail the fundamental formation mechanisms of different MXene-based hydrogels and their derivatives. Notably, MXenes can take multiple roles during the gelation process, from self-gelators to initiators, and from crosslinkers to nonparticipating nanofillers. Interestingly, the synergy between the properties of MXenes and the other elements in the hydrogel network has led to a plethora of advanced characteristics, including enhanced mechanical and electrochemical behavior, improved sensing properties, and biocompatibility. As a result, MXene-based hydrogels and their derivatives have demonstrated a phenomenal performance in a wide range of applications, spanning energy storage/harvesting, biomedical, catalysis, EMI shielding, and sensing.

Generally, an important attribute of gel chemistry is that minute changes in the interacting components can easily lead to variable functionalities. In the case of MXene-based hydrogels, the complex interplay between kinetics and thermodynamics of the gelation process is governed by various intermolecular interactions between the constituents of the 3D network. A deep understanding of these interactions is critical for developing MXene hydrogels with tailorable properties for particular applications. In this regard, systematic characterization (chemical and physical) protocols are in urgent need to grasp the micro/macroscopic behavior of MXene-based gels, as well as the underlying structure-property relationships.

In principle, analogous to other hydrogels, the gelation of MXene-based hydrogels embraces both covalent and noncovalent crosslinking. The former is typically strong and mostly static, whereas the latter is relatively weaker, allowing for dynamic assembly and disintegration. Although individual non-covalent interactions may not be strong, a large number of them can lead to a robust net interaction comparable to or even greater than covalent ones. The combinations of such diverse interactions between the multiscale elements in the MXene hydrogels provide ample opportunities for further innovations. It should also be noted that present studies are mostly oriented towards single applications. However, given the superior performance of MXene hydrogels in various applications, conducting molecular designs from a multifunctional perspective is appealing. Through careful design, multifunctional MXene hydrogels are expected to be useful for wearable/stretchable applications, such as an artificial skin that can sense and stimulate, or hydrogel materials with universal (e.g., strain, thermal, light, and ultrasonic) responsiveness.

On the other hand, the absence of studies of the fundamental issues such as mass/charge transport between MXene nanosheets and other components in the hydrogel system, kinetics/thermodynamics of the assembling process, and mechanism brought by the interactions between these constituents make the rational design of MXene hydrogels challenging. 
Theoretical and modeling studies concerning these aspects are still lacking and are urgently required. Moreover, presently, all reported MXene hydrogels and aerogels are mostly based on the $\mathrm{Ti}_{3} \mathrm{C}_{2} \mathrm{~T}_{x}$, i.e., the most mature member of the MXene family in terms of synthesis and investigated properties. Hence, given the available large number of carbide- and nitride-based MXenes with vastly different characteristics, there is a great room for further optimization of MXene-based hydrogels for target applications. To achieve this, however, more efficient MXene synthesis processes with better product control in terms of morphology, surface functionalization, sufficient crosslinking sites, nanosheet dimensions, etc. are needed. Furthermore, the practical applications of MXene hydrogels require well-developed scalable synthesis methods of MXenes, which is a further challenge given the inherent hazardousness of the used fluorine-based etchants. Thus, it is highly imperative to find less hazardous etchants that can result in the same product quality, yet with a higher yield.

Another important aspect to consider is that existing devices based on MXene hydrogels are mostly manufactured manually, which is laborious, time-consuming, hard to reproduce, or mass produce. To go beyond proof-of-concept device demonstrations, rapid prototyping methods which enable fast and low-cost mass production at millimeter and even sub-millimeter scale are urgently needed. There is no report as yet for patterning and shaping MXene hydrogels using traditional lithography-based methods. Not to mention advanced manufacturing techniques such as $3 \mathrm{D}$ printing, or $4 \mathrm{D}$ printing, which enables shapemorphing behaviors. To properly integrate MXene hydrogels into flexible, soft, wearable, and implantable devices, issues such as adhesion between hydrogels and elastomers or tissues need to be taken into account. For particular applications, the long-term stability of MXene hydrogels needs to be considered, especially when one considers water as an active environment, which can promote oxidation of MXene nanosheets. Furthermore, the drying out of hydrogels and the leaching of MXenes with time are likely to affect the stability and performance of the corresponding MXene hydrogels and may invoke environmental concerns. Hence, a careful device design is also required to tackle this issue.

\section{Conflicts of interest}

There are no conflicts to declare.

\section{Acknowledgements}

The research reported in this publication is supported by King Abdullah University of Science \& Technology (KAUST). The Natural Science Foundation of Jiangsu Province (BK20170999) and The National Natural Science Foundation of China (21805136) are also acknowledged.

\section{References}

1 T. Someya and M. Amagai, Nat. Biotechnol., 2019, 37, 382-388.
2 C. H. Yang and Z. G. Suo, Nat. Rev. Mater., 2018, 3, 125-142.

3 H. Yuk, B. Y. Lu and X. H. Zhao, Chem. Soc. Rev., 2019, 48, 1642-1667.

4 J. Zhang, Y. Hu and Y. Li, Gel Chemistry: Interactions, Structures and Properties, Springer, 2018.

5 S. Zhao, H.-B. Zhang, J.-Q. Luo, Q.-W. Wang, B. Xu, S. Hong and Z.-Z. Yu, ACS Nano, 2018, 12, 11193-11202.

6 X. Zhang, R. Lv, A. Wang, W. Guo, X. Liu and J. Luo, Angew. Chem., Int. Ed., 2018, 57, 15028-15033.

7 B. Anasori, M. R. Lukatskaya and Y. Gogotsi, Nat. Rev. Mater., 2017, 2, 1-17.

8 Y. Z. Zhang, Y. Wang, Q. Jiang, J. K. El-Demellawi, H. Kim and H. N. Alshareef, Adv. Mater., 2020, 1908486.

9 J. Pang, R. G. Mendes, A. Bachmatiuk, L. Zhao, H. Q. Ta, T. Gemming, H. Liu, Z. Liu and M. H. Rummeli, Chem. Soc. Rev., 2019, 48, 72-133.

10 Z. Fu, N. Wang, D. Legut, C. Si, Q. Zhang, S. Du, T. C. Germann, J. S. Francisco and R. Zhang, Chem. Rev., 2019, 119, 11980-12031.

11 K. Hantanasirisakul and Y. Gogotsi, Adv. Mater., 2018, 30, 1804779.

12 Z. Lin, J. Liu, W. Peng, Y. Zhu, Y. Zhao, K. Jiang, M. Peng and Y. Tan, ACS Nano, 2020, 14, 2109-2117.

13 Y.-Z. Zhang, K. H. Lee, D. H. Anjum, R. Sougrat, Q. Jiang, H. Kim and H. N. Alshareef, Sci. Adv., 2018, 4, eaat0098.

14 H. E. Karahan, K. Goh, C. Zhang, E. Yang, C. Yıldırım, C. Y. Chuah, M. G. Ahunbay, J. Lee, Ș. B. Tantekin-Ersolmaz and Y. Chen, Adv. Mater., 2020, 1906697.

15 Z. Wu, T. Shang, Y. Deng, Y. Tao and Q. H. Yang, Adv. Sci., 2020, 7, 1903077.

16 Y. Yue, N. Liu, Y. Ma, S. Wang, W. Liu, C. Luo, H. Zhang, F. Cheng, J. Rao, X. Hu, J. Su and Y. Gao, ACS Nano, 2018, 12, 4224-4232.

17 Y. Deng, T. Shang, Z. Wu, Y. Tao, C. Luo, J. Liang, D. Han, R. Lyu, C. Qi, W. Lv, F. Kang and Q.-H. Yang, Adv. Mater., 2019, 31, 1902432.

18 H. Liao, X. Guo, P. Wan and G. Yu, Adv. Funct. Mater., 2019, 29, 1904507.

19 K. H. Lee, Y.-Z. Zhang, Q. Jiang, H. Kim, A. A. Alkenawi and H. N. Alshareef, ACS Nano, 2020, 14, 3199-3207.

20 M. Alhabeb, K. Maleski, B. Anasori, P. Lelyukh, L. Clark, S. Sin and Y. Gogotsi, Chem. Mater., 2017, 29, 7633-7644.

21 G. Ye, Z. Wen, F. Wen, X. Song, L. Wang, C. Li, Y. He, S. Prakash and X. Qiu, Theranostics, 2020, 10, 2047-2066.

22 Z. Lin, D. Barbara, P.-L. Taberna, K. L. Van Aken, B. Anasori, Y. Gogotsi and P. Simon, J. Power Sources, 2016, 326, 575-579.

23 M. R. Lukatskaya, S. Kota, Z. Lin, M.-Q. Zhao, N. Shpigel, M. D. Levi, J. Halim, P.-L. Taberna, M. W. Barsoum, P. Simon and Y. Gogotsi, Nat. Energy, 2017, 2, 17105.

24 E. Kayali, A. VahidMohammadi, J. Orangi and M. Beidaghi, ACS Appl. Mater. Interfaces, 2018, 10, 25949-25954.

25 P. Lin, J. Xie, Y. He, X. Lu, W. Li, J. Fang, S. Yan, L. Zhang, X. Sheng and Y. Chen, Sol. Energy Mater. Sol. Cells, 2020, 206, 110229.

26 Y. Chen, X. Xie, X. Xin, Z.-R. Tang and Y.-J. Xu, ACS Nano, 2018, 13, 295-304. 
27 X. Xie, Z. Wu and N. Zhang, Chin. Chem. Lett., 2020, 31, 1014-1017.

28 C. Yang, Q. Jiang, W. Li, H. He, L. Yang, Z. Lu and H. Huang, Chem. Mater., 2019, 31, 9277-9287.

29 T. Shang, Z. Lin, C. Qi, X. Liu, P. Li, Y. Tao, Z. Wu, D. Li, P. Simon and Q.-H. Yang, Adv. Funct. Mater., 2019, 29, 1903960.

30 C. Xing, S. Chen, X. Liang, Q. Liu, M. Qu, Q. Zou, J. Li, H. Tan, L. Liu, D. Fan and H. Zhang, ACS Appl. Mater. Interfaces, 2018, 10, 27631-27643.

31 P. Zhang, X.-J. Yang, P. Li, Y. Zhao and Q. J. Niu, Soft Matter, 2020, 16, 162-169.

32 N. Tao, D. Zhang, X. Li, D. Lou, X. Sun, C. Wei, J. Li, J. Yang and Y.-N. Liu, Chem. Sci., 2019, 10, 10765-10771.

33 J. Zhang, L. Wan, Y. Gao, X. Fang, T. Lu, L. Pan and F. Xuan, Adv. Electron. Mater., 2019, 5, 1900285.

34 A. Rafieerad, G. L. Sequiera, W. Yan, P. Kaur, A. Amiri and S. Dhingra, J. Mech. Behav. Biomed. Mater., 2020, 101, 103440.

35 Z. Chen, Y. Hu, H. Zhuo, L. Liu, S. Jing, L. Zhong, X. Peng and R.-C. Sun, Chem. Mater., 2019, 31, 3301-3312.

36 Y. Hu, H. Zhuo, Q. Luo, Y. Wu, R. Wen, Z. Chen, L. Liu, L. Zhong, X. Peng and R. Sun, J. Mater. Chem. A, 2019, 7, 10273-10281.

37 Z. Fan, D. Wang, Y. Yuan, Y. Wang, Z. Cheng, Y. Liu and Z. Xie, Chem. Eng. J., 2020, 381, 122696.

38 J. Liu, H. B. Zhang, X. Xie, R. Yang, Z. Liu, Y. Liu and Z. Z. Yu, Small, 2018, 14, 1802479.

39 Y. Ma, Y. Yue, H. Zhang, F. Cheng, W. Zhao, J. Rao, S. Luo, J. Wang, X. Jiang, Z. Liu, N. Liu and Y. Gao, ACS Nano, 2018, 12, 3209-3216.

40 N. N. Wang, H. Wang, Y. Y. Wang, Y. H. Wei, J. Y. Si, A. C. Y. Yuen, J. S. Xie, B. Yu, S. E. Zhu, H. D. Lu, W. Yang, Q. N. Chan and G. H. Yeoh, ACS Appl. Mater. Interfaces, 2019, 11, 40512-40523.
41 M. Zhu, Y. Yue, Y. Cheng, Y. Zhang, J. Su, F. Long, X. Jiang, Y. Ma and Y. Gao, Adv. Electron. Mater., 2019, 6, 1901064.

42 H. Xu, X. Yin, X. Li, M. Li, S. Liang, L. Zhang and L. Cheng, ACS Appl. Mater. Interfaces, 2019, 11, 10198-10207.

43 Y. Jiang, X. Xie, Y. Chen, Y. Liu, R. Yang and G. Sui, J. Mater. Chem. C, 2018, 6, 8679-8687.

44 X. Zhao, L.-M. Peng, C.-Y. Tang, J.-H. Pu, X.-J. Zha, K. Ke, R.-Y. Bao, M.-B. Yang and W. Yang, Mater. Horiz., 2020, 7, 855-865.

45 J. Song, X. Guo, J. Zhang, Y. Chen, C. Zhang, L. Luo, F. Wang and G. Wang, J. Mater. Chem. A, 2019, 7, 6507-6513.

46 Q. Wang, S. Wang, X. Guo, L. Ruan, N. Wei, Y. Ma, J. Li, M. Wang, W. Li and W. Zeng, Adv. Electron. Mater., 2019, 5, 1900537.

47 Z. Zhou, J. Liu, X. Zhang, D. Tian, Z. Zhan and C. Lu, Adv. Mater. Interfaces, 2019, 6, 1802040.

48 H. Zhang, P. Zhang, W. Zheng, W. Tian, J. Chen, Y. Zhang and Z. Sun, Electrochim. Acta, 2018, 285, 94-102.

49 Y. Ma, N. Liu, L. Li, X. Hu, Z. Zou, J. Wang, S. Luo and Y. Gao, Nat. Commun., 2017, 8, 1-8.

50 J. K. El-Demellawi, S. Lopatin, J. Yin, O. F. Mohammed and H. N. Alshareef, ACS Nano, 2018, 12, 8485-8493.

51 B. Escuder and J. F. Miravet, Functional molecular gels, Royal Society of Chemistry, 2013.

52 G. Liu, J. Zou, Q. Tang, X. Yang, Y. Zhang, Q. Zhang, W. Huang, P. Chen, J. Shao and X. Dong, ACS Appl. Mater. Interfaces, 2017, 9, 40077-40086.

53 G. Ge, W. Yuan, W. Zhao, Y. Lu, Y. Zhang, W. Wang, P. Chen, W. Huang, W. Si and X. Dong, J. Mater. Chem. A, 2019, 7, 5949-5956.

54 G. Ge, Y. Lu, X. Qu, W. Zhao, Y. Ren, W. Wang, Q. Wang, W. Huang and X. Dong, ACS Nano, 2019, 14, 218-228.

55 R. Hinchet, H.-J. Yoon, H. Ryu, M.-K. Kim, E.-K. Choi, D.-S. Kim and S.-W. Kim, Science, 2019, 365, 491-494. 